\title{
Interval 2-Tuple Linguistic Distance Operators and Their Applications to Supplier Evaluation and Selection
}

\author{
Meng-Meng Shan, ${ }^{1}$ Ping Li, ${ }^{2}$ and Hu-Chen Liu ${ }^{1,3}$ \\ ${ }^{1}$ School of Management, Shanghai University, Shanghai 200444, China \\ ${ }^{2}$ Zhoupu Hospital Affiliated to Shanghai University of Medicine \& Health Sciences, Shanghai 201318, China \\ ${ }^{3}$ School of Economics and Management, Tongji University, Shanghai 200092, China \\ Correspondence should be addressed to Hu-Chen Liu; huchenliu@foxmail.com
}

Received 11 August 2016; Revised 28 October 2016; Accepted 1 November 2016

Academic Editor: Anna M. Gil-Lafuente

Copyright (C) 2016 Meng-Meng Shan et al. This is an open access article distributed under the Creative Commons Attribution License, which permits unrestricted use, distribution, and reproduction in any medium, provided the original work is properly cited.

With respect to multicriteria supplier selection problems with interval 2-tuple linguistic information, a new decision making approach that uses distance measures is proposed. Motivated by the ordered weighted distance (OWD) measures, in this paper, we develop some interval 2-tuple linguistic distance operators such as the interval 2-tuple weighted distance (ITWD), the interval 2-tuple ordered weighted distance (ITOWD), and the interval 2-tuple hybrid weighted distance (ITHWD) operators. These aggregation operators are very useful for the treatment of input data in the form of interval 2-tuple linguistic variables. We study some desirable properties of the ITOWD operator and further generalize it by using the generalized and the quasi-arithmetic means. Finally, the new approach is utilized to complete a supplier selection study for an actual hospital from the healthcare industry.

\section{Introduction}

The ordered weighted averaging (OWA) operator [1] is a very well-known aggregation operator, providing a parameterized family of aggregation operators which includes the maximum, the minimum, and the average. The prominent characteristic of the OWA operator is the reordering step. An interesting extension of the OWA is the use of distance measures in the OWA operator. In this respect, $\mathrm{Xu}$ and Chen [2] developed the ordered weighted distance (OWD) measure, which is the generalization of a variety of wellknown distance measures, such as the normalized Hamming distance, the normalized Euclidean distance, and the normalized geometric distance. The prominent characteristic of the OWD measure is that it can relieve (or intensify) the influence of unduly large or unduly small deviations on the aggregation results by assigning them low (or high) weights. Merigó and Gil-Lafuente [3] proposed a technique for decision making using the OWA operator to calculate Hamming distance and introduced the ordered weighted averaging distance (OWAD) operator. The main advantage of this operator is that it can take into account the attitudinal character of a decision maker in the aggregation process; thus the decision maker is able to consider the decision problem more clearly according to his or her interests. Merigó et al. [4] introduced the probabilistic ordered weighted averaging distance (POWAD) operator, which uses a unified model between the probability and the OWA operator considering the degree of importance that each concept has in the aggregation. Zeng et al. [5] further extended the POWAD operator to deal with uncertain environments represented in the form of interval numbers and proposed the uncertain probabilistic ordered weighted averaging distance (UPOWAD) operator. Merigó et al. [6] studied the use of distance measures and heavy aggregations in the OWA operator and presented the heavy ordered weighted averaging distance (HOWAD) operator. It is a new aggregation operator that provides a parameterized family of aggregation operators between the minimum distance and the total distance operator.

In addition, Merigó and Casanovas [7] introduced the linguistic ordered weighted averaging distance (LOWAD) operator for linguistic decision making. Zeng and Su [8] and 
Zeng [9] considered the situations with intuitionistic fuzzy and interval-valued intuitionistic information and developed some intuitionistic fuzzy weighted distance measures. Xu [10] developed some fuzzy ordered distance measures for group decision making with linguistic, interval, triangular, or trapezoidal fuzzy preference information. Xian and Sun [11] developed the fuzzy linguistic induced Euclidean ordered weighted averaging distance (FLIEOWAD) operator for group linguistic decision making, in which the criteria values take the form of fuzzy linguistic information. More recently, other different types of distance measures have been proposed in the literature, like the uncertain induced heavy ordered weighted averaging distance (UIHOWAD) operator [12], the continuous intuitionistic fuzzy ordered weighted distance (C-IFOWD) measure [13], the Pythagorean fuzzy ordered weighted averaging weighted average distance (PFOWAWAD) operator [14], the fuzzy linguistic induced ordered weighted averaging Minkowski distance (FLIOWAMD) operator [15], the generalized interval-valued 2-tuple linguistic weighted distance measures [16], the generalized hesitant fuzzy linguistic weighted distance measures [17], and so on [18-21].

In many situations, however, the input arguments may take the form of interval 2-tuple linguistic variables [2527] because of time pressure, lack of knowledge or data, and decision makers' limited attention and information processing capabilities. Furthermore, decision makers may use different linguistic term sets to express their evaluations on the established selection criteria considering their personal backgrounds, preferences, and different understanding levels to the alternatives. Therefore, it is necessary to extend the ordered weighted distance measures to accommodate the interval 2-tuple linguistic environment [28-30], which is also the focus of this paper. For doing so, we will develop some interval 2-tuple linguistic distance operators such as interval 2-tuple weighted distance (ITWD), interval 2-tuple ordered weighted distance (ITOWD), and interval 2-tuple hybrid weighted distance (ITHWD) operators. These aggregation operators are very effective to deal with situations where the input data are expressed in interval 2-tuple linguistic variables. We study some desirable properties of the ITOWD operator and further generalize it by using the generalized and the quasi-arithmetic means obtaining the generalized interval 2-tuple ordered weighted distance (GITOWD) and the quasi-arithmetic interval 2-tuple ordered weighted distance (Quasi-ITOWD) operators. Finally, based on the GITOWD operator, we develop an approach to group supplier evaluation and selection with interval 2-tuple linguistic information and illustrate it with a numerical example.

The remainder of this paper is set out as follows. In Section 2, we introduce some basic concepts and operation laws of interval 2-tuple linguistic variables. In Section 3, we develop the ITWD, the ITOWD, and the ITHWD operators and investigate some desirable properties of the ITOWD operator. In Section 4, we present an approach based on the developed interval 2-tuple linguistic distance operators to multicriteria group supplier selection. A supplier selection example is given in Section 5 to verify the proposed approach and to demonstrate its feasibility and practicality. Finally, conclusions and future directions are provided in Section 6.

\section{Preliminaries}

2.1. 2-Tuple Linguistic Variables. The 2-tuple linguistic representation model was firstly presented in [31] based on the concept of symbolic translation. It is used to represent linguistic information by means of a linguistic 2-tuple, $(s, \alpha)$, where $s$ is a linguistic term from the predefined linguistic term set $S$ and $\alpha$ is a numerical value representing the symbolic translation. In the classical 2-tuple linguistic approach, the range of $\beta$ is between 0 and $g$, which is relevant to the granularity of a linguistic term set. Here, $\beta$ is the result of an aggregation of the indices of a set of labels assessed in the linguistic term set $S$. To overcome this restriction, Tai and Chen [32] proposed a generalized 2-tuple linguistic model and translation functions.

Definition 1. Let $S=\left\{s_{0}, s_{1}, \ldots, s_{g}\right\}$ be a linguistic term set and let $\beta \in[0,1]$ be a value representing the result of a symbolic aggregation operation. Then the generalized translation function $\Delta$ used to obtain the 2-tuple linguistic variable equivalent to $\beta$ is defined as follows [32]:

$$
\begin{aligned}
\Delta:[0,1] & \longrightarrow S \times\left[-\frac{1}{2 g}, \frac{1}{2 g}\right), \\
\Delta(\beta)=\left(s_{i}, \alpha\right), & \text { with } \begin{cases}s_{i}, & i=\operatorname{round}(\beta \cdot g) \\
\alpha=\beta-\frac{i}{g}, & \alpha \in\left[-\frac{1}{2 g}, \frac{1}{2 g}\right),\end{cases}
\end{aligned}
$$

where round $(\cdot)$ is the usual rounding operation, $s_{i}$ has the closest index label to $\beta$, and $\alpha$ is the value of the symbolic translation.

Definition 2. Let $S=\left\{s_{0}, s_{1}, \ldots, s_{g}\right\}$ be a linguistic term set and let $\left(s_{i}, \alpha\right)$ be a 2 -tuple. There exists a function $\Delta^{-1}$ which is able to convert a 2-tuple linguistic variable into its equivalent numerical value $\beta \in[0,1]$. The reverse function $\Delta^{-1}$ is defined as follows [32]:

$$
\begin{aligned}
\Delta^{-1}: S \times\left[-\frac{1}{2 g}, \frac{1}{2 g}\right) & \longrightarrow[0,1], \\
\Delta^{-1}\left(s_{i}, \alpha\right) & =\frac{i}{g}+\alpha=\beta .
\end{aligned}
$$

Particularly, it is necessary to point out that the conversion of a linguistic term into a linguistic 2-tuple consists of adding a value 0 as symbolic translation [31]:

$$
s_{i} \in S \Longrightarrow\left(s_{i}, 0\right) \text {. }
$$

The comparison of linguistic information represented by 2-tuples is carried out according to an ordinary lexicographic order.

Definition 3. Let $\left(s_{k}, \alpha_{1}\right)$ and $\left(s_{l}, \alpha_{2}\right)$ be two 2-tuples; then [31, 33]

(1) if $k<l$, then $\left(s_{k}, \alpha_{1}\right)$ is smaller than $\left(s_{l}, \alpha_{2}\right)$; 
(2) if $k=l$, then one has the following:

(a) if $\alpha_{1}=\alpha_{2}$, then $\left(s_{k}, \alpha_{1}\right)$ is equal to $\left(s_{l}, \alpha_{2}\right)$;

(b) if $\alpha_{1}<\alpha_{2}$, then $\left(s_{k}, \alpha_{1}\right)$ is smaller than $\left(s_{l}, \alpha_{2}\right)$;

(c) if $\alpha_{1}>\alpha_{2}$, then $\left(s_{k}, \alpha_{1}\right)$ is bigger than $\left(s_{l}, \alpha_{2}\right)$.

2.2. Interval 2-Tuple Linguistic Variables. Motivated by Xu's uncertain linguistic variables [22] and based on the definitions of [32], Zhang [34] proposed the interval 2-tuple linguistic representation model as generalization of the 2tuple linguistic variables. Due to its characteristics and advantages, the interval 2-tuple linguistic representation model has been widely applied for dealing with uncertainty in various multicriteria decision making problems [35-38]. It can be defined as follows.

Definition 4. Let $S=\left\{s_{0}, s_{1}, \ldots, s_{g}\right\}$ be a linguistic term set. An interval 2-tuple linguistic variable is composed of two 2-tuples, denoted by $\left[\left(s_{i}, \alpha_{1}\right),\left(s_{j}, \alpha_{2}\right)\right]$, where $\left(s_{i}, \alpha_{1}\right) \leq$ $\left(s_{j}, \alpha_{2}\right), s_{i}\left(s_{j}\right)$, and $\alpha_{1}\left(\alpha_{2}\right)$ represent the linguistic label of $S$ and symbolic translation, respectively. The interval 2-tuple that expresses the equivalent information to an interval value $\left[\beta_{1}, \beta_{2}\right]\left(\beta_{1}, \beta_{2} \in[0,1], \beta_{1} \leq \beta_{2}\right)$ is derived by the following function $[25,34]$ :

$$
\begin{aligned}
& \Delta\left[\beta_{1}, \beta_{2}\right]=\left[\left(s_{i}, \alpha_{1}\right),\left(s_{j}, \alpha_{2}\right)\right] \\
& \text { with } \begin{cases}s_{i}, & i=\operatorname{round}\left(\beta_{1} \cdot g\right) \\
s_{j}, & j=\operatorname{round}\left(\beta_{2} \cdot g\right) \\
\alpha_{1}=\beta_{1}-\frac{i}{g}, & \alpha_{1} \in\left[-\frac{1}{2 g}, \frac{1}{2 g}\right) \\
\alpha_{2}=\beta_{2}-\frac{j}{g}, & \alpha_{2} \in\left[-\frac{1}{2 g}, \frac{1}{2 g}\right) .\end{cases}
\end{aligned}
$$

On the contrary, there is always a function $\Delta^{-1}$ such that an interval 2-tuple can be converted into an interval value $\left[\beta_{1}, \beta_{2}\right]\left(\beta_{1}, \beta_{2} \in[0,1], \beta_{1} \leq \beta_{2}\right)$ as follows:

$$
\Delta^{-1}\left[\left(s_{i}, \alpha_{1}\right),\left(s_{j}, \alpha_{2}\right)\right]=\left[\frac{i}{g}+\alpha_{1}, \frac{j}{g}+\alpha_{2}\right]=\left[\beta_{1}, \beta_{2}\right] .
$$

In particular, if $s_{i}=s_{j}$ and $\alpha_{1}=\alpha_{2}$, then the interval 2-tuple linguistic variable reduces to a 2 -tuple linguistic variable.

Note that the uncertain linguistic variable [32] is simpler than the interval 2-tuple linguistic variable, which can also be used for dealing with group decision making with uncertain linguistic information. However, compared with the uncertain linguistic variables, Zhang's interval 2-tuple linguistic variables have the following advantages [31, 34, 39]. (1) The interval 2-tuple linguistic variable has exact characteristic in linguistic information processing, which can effectively avoid information distortion and loss in the linguistic information processing. In contrast, the uncertain linguistic variable performs the retranslation step as an approximation process to express the results in the original expression domain (initial discrete linguistic term set) provoking a lack of accuracy. (2)
In the process of aggregating uncertain linguistic information, the operational laws of uncertain linguistic variables are not closed. Let $S=\left\{s_{0}, s_{1}, \ldots, s_{6}\right\}$ be a linguistic term set; we have $\left[s_{2}, s_{3}\right] \oplus\left[s_{4}, s_{5}\right]=\left[s_{6}, s_{8}\right]$ and $\left[s_{4}, s_{5}\right] \otimes\left[s_{2}, s_{3}\right]=$ $\left[s_{8}, s_{15}\right]$. Clearly, the results of operation exceed the range of the linguistic term set $S$. This problem is solved by employing the interval 2-tuple linguistic variables. (3) Decision makers can express their preferences by the use of linguistic term sets with different granularity of uncertainty and their judgments can be better expressed with interval 2-tuples from the preestablished linguistic term sets. But the uncertain linguistic variables may lead to inflexibility for managing the group decision making problem with multigranularity linguistic information.

Based on the operations of uncertain linguistic variables [22], Zhang [34] further gave some basic operational laws of interval 2-tuples and proposed the interval 2-tuple weighted average (ITWA) operator.

Definition 5. Consider any three interval 2-tuples, $\tilde{a}=$ $[(r, \alpha),(t, \varepsilon)], \widetilde{a}_{1}=\left[\left(r_{1}, \alpha_{1}\right),\left(t_{1}, \varepsilon_{1}\right)\right]$, and $\widetilde{a}_{2}=\left[\left(r_{2}, \alpha_{2}\right),\left(t_{2}\right.\right.$, $\left.\left.\varepsilon_{2}\right)\right]$, and let $\lambda \in[0,1]$; then their operations are defined as follows [34]:

(1) $\tilde{a}_{1} \oplus \tilde{a}_{2}=\left[\left(r_{1}, \alpha_{1}\right),\left(t_{1}, \varepsilon_{1}\right)\right] \oplus\left[\left(r_{2}, \alpha_{2}\right),\left(t_{2}, \varepsilon_{2}\right)\right]=$ $\Delta\left[\Delta^{-1}\left(r_{1}, \alpha_{1}\right)+\Delta^{-1}\left(r_{2}, \alpha_{2}\right), \Delta^{-1}\left(t_{1}, \varepsilon_{1}\right)+\Delta^{-1}\left(t_{2}, \varepsilon_{2}\right)\right] ;$

(2) $\lambda \widetilde{a}=\lambda[(r, \alpha),(t, \varepsilon)]=\Delta\left[\lambda \Delta^{-1}(r, \alpha), \lambda \Delta^{-1}(t, \varepsilon)\right]$.

Definition 6. Let $\tilde{a}_{i}=\left[\left(r_{i}, \alpha_{i}\right),\left(t_{i}, \varepsilon_{i}\right)\right](i=1,2, \ldots, n)$ be a set of interval 2-tuples and let $w=\left(w_{1}, w_{2}, \ldots, w_{n}\right)^{T}$ be their associated weights, with $w_{i} \in[0,1]$ and $\sum_{i=1}^{n} w_{i}=1$. The ITWA operator is defined as [34]

$$
\begin{aligned}
& \operatorname{ITWA}_{w}\left(\tilde{a}_{1}, \tilde{a}_{2}, \ldots, \tilde{a}_{n}\right)=\bigoplus_{i=1}^{n}\left(w_{i} \tilde{a}_{i}\right) \\
& =\Delta\left[\sum_{i=1}^{n} w_{i} \Delta^{-1}\left(r_{i}, \alpha_{i}\right), \sum_{i=1}^{n} w_{i} \Delta^{-1}\left(t_{i}, \varepsilon_{i}\right)\right] .
\end{aligned}
$$

Inspired by the distance measure in uncertain linguistic environment [40], the distance between interval 2-tuples can be defined below.

Definition 7. Let $\tilde{a}_{1}=\left[\left(r_{1}, \alpha_{1}\right),\left(t_{1}, \varepsilon_{1}\right)\right]$ and $\tilde{a}_{2}=\left[\left(r_{2}, \alpha_{2}\right),\left(t_{2}\right.\right.$, $\left.\varepsilon_{2}\right)$ ] be any two interval 2-tuples; then

$$
\begin{aligned}
& d_{\mathrm{ITD}}(\tilde{a}, \tilde{b})=\Delta\left[\frac { 1 } { 2 } \left(\left|\Delta^{-1}\left(r_{1}, \alpha_{1}\right)-\Delta^{-1}\left(r_{2}, \alpha_{2}\right)\right|\right.\right. \\
& \left.\left.+\left|\Delta^{-1}\left(t_{1}, \varepsilon_{1}\right)-\Delta^{-1}\left(t_{2}, \varepsilon_{2}\right)\right|\right)\right]
\end{aligned}
$$

is called the interval 2-tuple distance between $\tilde{a}$ and $\tilde{b}$. Particularly, if the interval 2-tuples $\widetilde{a}_{1}=\left[\left(r_{1}, \alpha_{1}\right),\left(t_{1}, \varepsilon_{1}\right)\right]$ and $\widetilde{a}_{2}=$ $\left[\left(r_{2}, \alpha_{2}\right),\left(t_{2}, \varepsilon_{2}\right)\right]$ are degenerated to 2 -tuples $\widehat{a}=\left(r_{1}, \alpha_{1}\right)$ and $\widehat{b}=\left(r_{2}, \alpha_{2}\right)$, then the interval 2-tuple distance will become the 2-tuple distance [41]. 


\section{Interval 2-Tuple Linguistic Distance Operators}

The ordered weighted averaging distance (OWAD) operator [3] is an extension of the traditional Hamming distance by using the OWA operator, which provides a parameterized family of aggregation operators ranging from the minimum to the maximum distance. For two real numbers sets $A=$ $\left\{a_{1}, a_{2}, \ldots, a_{n}\right\}$ and $B=\left\{b_{1}, b_{2}, \ldots, b_{n}\right\}$, the OWAD operator is defined as follows.

Definition 8. An OWAD operator of dimension $n$ is a mapping OWAD: $R^{n} \times R^{n} \rightarrow R$ which has an associated weighting vector $\omega=\left(\omega_{1}, \omega_{2}, \ldots, \omega_{n}\right)^{T}$, with $\omega_{j} \in[0,1]$ and $\sum_{j=1}^{n} \omega_{j}=1$, such that

$$
\operatorname{OWAD}\left(\left\langle a_{1}, b_{1}\right\rangle,\left\langle a_{2}, b_{2}\right\rangle, \ldots,\left\langle a_{n}, b_{n}\right\rangle\right)=\sum_{j=1}^{n} \omega_{j} d_{j},
$$

where $d_{j}$ represents the $j$ th largest of the individual distance $\left|a_{i}-b_{i}\right|$.

Further, $\mathrm{Xu}$ and Chen [2] developed an ordered weighted distance (OWD) measure, which generalizes a variety of wellknown distance measures and aggregation operators.

Definition 9. An OWD measure of dimension $n$ is a mapping OWD: $R^{n} \times R^{n} \rightarrow R$ which has an associated weighting vector $\omega=\left(\omega_{1}, \omega_{2}, \ldots, \omega_{n}\right)^{T}$, with $\omega_{j} \in[0,1]$ and $\sum_{j=1}^{n} \omega_{j}=1$, such that

$$
\begin{aligned}
\operatorname{OWD} & \left(\left\langle a_{1}, b_{1}\right\rangle,\left\langle a_{2}, b_{2}\right\rangle, \ldots,\left\langle a_{n}, b_{n}\right\rangle\right) \\
= & \left(\sum_{j=1}^{n} \omega_{j} d_{j}^{\lambda}\right)^{1 / \lambda},
\end{aligned}
$$

where $d_{j}$ is the $j$ th largest of the individual distance $\mid a_{i}-$ $b_{i} \mid$. If $\lambda=1$, then the OWD measure is reduced to the OWAD operator; if $\lambda=1$ and $\omega=(1 / n, 1 / n, \ldots, 1 / n)^{T}$, then the OWD measure is reduced to the normalized Hamming distance.

3.1. Interval 2-Tuple Linguistic Distance Operators. The OWAD and the OWD operators have only been used in the situations in which the input arguments are exact values. However, judgments of people depend on personal psychological aspects such as experience, learning, situation, and state of mind. It is more suitable for decision makers to provide their preferences by means of linguistic variables rather than numerical ones.

For convenience, let $\widetilde{S}$ be the set of all interval 2-tuples, let $\widehat{S}$ be the set of all 2-tuples, and let $\widetilde{A}=\left\{\widetilde{a}_{1}, \widetilde{a}_{2}, \ldots, \widetilde{a}_{n}\right\}$ and $\widetilde{B}=\left\{\widetilde{b}_{1}, \widetilde{b}_{2}, \ldots, \widetilde{b}_{n}\right\}$ be two sets of interval 2-tuples. Based on (7), we define an interval 2-tuple weighted distance (ITWD) operator as follows.

Definition 10. An ITWD operator of dimension $n$ is a mapping ITWD: $\widetilde{S}^{n} \times \widetilde{S}^{n} \rightarrow \widehat{S}$, which has an associated weight vector $w=\left(w_{1}, w_{2}, \ldots, w_{n}\right)^{T}$ with $w_{i} \in[0,1]$ and $\sum_{i=1}^{n} w_{i}=1$, such that

$$
\begin{aligned}
& \operatorname{ITWD}\left(\left\langle\widetilde{a}_{1}, \tilde{b}_{1}\right\rangle,\left\langle\widetilde{a}_{2}, \tilde{b}_{2}\right\rangle, \ldots,\left\langle\widetilde{a}_{n}, \widetilde{b}_{n}\right\rangle\right) \\
& =\sum_{i=1}^{n} w_{i} d_{\operatorname{ITD}}\left(\tilde{a}_{i}, \tilde{b}_{i}\right)
\end{aligned}
$$

where $d_{\text {ITD }}\left(\widetilde{a}_{i}, \widetilde{b}_{i}\right)$ is the interval 2-tuple distance between $\tilde{a}_{i}$ and $\widetilde{b}_{i}$.

In particular, if $w=(1 / n, 1 / n, \ldots, 1 / n)^{T}$, then the ITWD becomes the interval 2-tuple normalized distance (ITND) operator:

$$
\begin{aligned}
& \operatorname{ITND}\left(\left\langle\tilde{a}_{1}, \widetilde{b}_{1}\right\rangle,\left\langle\tilde{a}_{2}, \widetilde{b}_{2}\right\rangle, \ldots,\left\langle\widetilde{a}_{n}, \tilde{b}_{n}\right\rangle\right) \\
& \quad=\frac{1}{n} \sum_{i=1}^{n} d_{\text {ITD }}\left(\tilde{a}_{i}, \widetilde{b}_{i}\right) .
\end{aligned}
$$

If the sets of interval 2-tuples $\widetilde{A}=\left\{\widetilde{a}_{1}, \widetilde{a}_{2}, \ldots, \widetilde{a}_{n}\right\}$ and $\widetilde{B}=\left\{\widetilde{b}_{1}, \widetilde{b}_{2}, \ldots, \widetilde{b}_{n}\right\}$ are degenerated to the sets of 2-tuples $\widehat{A}=\left\{\widehat{a}_{1}, \widehat{a}_{2}, \ldots, \widehat{a}_{n}\right\}$ and $\widehat{B}=\left\{\widehat{b}_{1}, \widehat{b}_{2}, \ldots, \widehat{b}_{n}\right\}$, then the ITWD is reduced to the 2-tuple weighted distance (TWD) operator:

$$
\begin{aligned}
& \operatorname{TWD}\left(\left\langle\widehat{a}_{1}, \widehat{b}_{1}\right\rangle,\left\langle\hat{a}_{2}, \widehat{b}_{2}\right\rangle, \ldots,\left\langle\widehat{a}_{n}, \widehat{b}_{n}\right\rangle\right) \\
& =\sum_{i=1}^{n} w_{i} d_{\mathrm{TD}}\left(\widehat{a}_{i}, \widehat{b}_{i}\right),
\end{aligned}
$$

where $d_{\mathrm{TD}}\left(\widehat{a}_{i}, \widehat{b}_{i}\right)$ is the 2-tuple distance between $\widehat{a}_{i}$ and $\widehat{b}_{i}$.

Based on the OWA and the ITWD operators, we define an interval 2-tuple ordered weighted distance (ITOWD) operator as follows.

Definition 11. An ITOWD operator of dimension $n$ is a mapping ITOWD: $\widetilde{S}^{n} \times \widetilde{S}^{n} \rightarrow \widehat{S}$, which has an associated weight vector $\omega=\left(\omega_{1}, \omega_{2}, \ldots, \omega_{n}\right)^{T}$, with $\omega_{j} \in[0,1]$ and $\sum_{j=1}^{n} \omega_{j}=1$, such that

$$
\begin{aligned}
& \operatorname{ITOWD}\left(\left\langle\tilde{a}_{1}, \tilde{b}_{1}\right\rangle,\left\langle\tilde{a}_{2}, \tilde{b}_{2}\right\rangle, \ldots,\left\langle\tilde{a}_{n}, \tilde{b}_{n}\right\rangle\right) \\
& =\sum_{j=1}^{n} \omega_{j} d_{\text {ITD }}\left(\tilde{a}_{\sigma(j)}, \tilde{b}_{\sigma(j)}\right),
\end{aligned}
$$

where $d_{\text {ITD }}\left(\widetilde{a}_{\sigma(j)}, \widetilde{b}_{\sigma(j)}\right)$ is the $j$ th largest of the interval 2-tuple distance $d_{\mathrm{ITD}}\left(\widetilde{a}_{i}, \widetilde{b}_{i}\right)$.

Particularly, if there is a tie between $d_{\mathrm{ITD}}\left(\widetilde{a}_{i}, \widetilde{b}_{i}\right)$ and $d_{\text {ITD }}\left(\widetilde{a}_{j}, \widetilde{b}_{j}\right)$, then we replace each of $d_{\text {ITD }}\left(\widetilde{a}_{i}, \widetilde{b}_{i}\right)$ and $d_{\text {ITD }}\left(\widetilde{a}_{j}, \widetilde{b}_{j}\right)$ by their average $\left(d_{\text {ITD }}\left(\widetilde{a}_{i}, \widetilde{b}_{i}\right)+d_{\text {ITD }}\left(\widetilde{a}_{j}, \widetilde{b}_{j}\right)\right) / 2$ in the process of aggregation. If $k$ items are tied, then we replace these by $k$ replicas of their average. If $\omega=(1 / n, 1 / n, \ldots, 1 / n)^{T}$, then the ITOWD becomes the ITND; if the position of $d_{\mathrm{ITD}}\left(\widetilde{a}_{i}, \widetilde{b}_{i}\right)$ is the same as the ordered position of $d_{\text {ITD }}\left(\widetilde{a}_{\sigma(j)}, \widetilde{b}_{\sigma(j)}\right)$, then the ITWD is obtained. Moreover, if $\widetilde{A}$ and $\widetilde{B}$ are degenerated to $\widehat{A}$ and $\widehat{B}$, then the ITOWD is 
reduced to the 2-tuple ordered weighted distance (TOWD) operator:

$$
\begin{aligned}
& \operatorname{TOWD}\left(\left\langle\widehat{a}_{1}, \widehat{b}_{1}\right\rangle,\left\langle\widehat{a}_{2}, \widehat{b}_{2}\right\rangle, \ldots,\left\langle\widehat{a}_{n}, \widehat{b}_{n}\right\rangle\right) \\
& =\sum_{j=1}^{n} \omega_{j} d_{\mathrm{TD}}\left(\widehat{a}_{\sigma(j)}, \widehat{b}_{\sigma(j)}\right)
\end{aligned}
$$

where $d_{\mathrm{TD}}\left(\widehat{a}_{\sigma(j)}, \widehat{b}_{\sigma(j)}\right)$ is the $j$ th largest of the 2 -tuple distance $d_{\mathrm{TD}}\left(\widehat{a}_{i}, \widehat{b}_{i}\right)$.

Similar to the OWAD operator, the ITOWD operator is commutative, monotonic, idempotent, and bounded. These properties can be shown with the following theorems.

Theorem 12 (commutativity-OWA aggregation). Assume that $f$ is the ITOWD operator. If $\left(\left\langle\tilde{a}_{1}^{\prime}, \widetilde{b}_{1}^{\prime}\right\rangle,\left\langle\tilde{a}_{2}^{\prime}, \widetilde{b}_{2}^{\prime}\right\rangle, \ldots\right.$, $\left.\left\langle\widetilde{a}_{n}^{\prime}, \widetilde{b}_{n}^{\prime}\right\rangle\right)$ is any permutation of the arguments $\left(\left\langle\widetilde{a}_{1}, \widetilde{b}_{1}\right\rangle,\left\langle\widetilde{a}_{2}\right.\right.$, $\left.\left.\widetilde{b}_{2}\right\rangle, \ldots,\left\langle\widetilde{a}_{n}, \widetilde{b}_{n}\right\rangle\right)$, then

$$
\begin{aligned}
f & \left(\left\langle\widetilde{a}_{1}, \widetilde{b}_{1}\right\rangle,\left\langle\widetilde{a}_{2}, \widetilde{b}_{2}\right\rangle, \ldots,\left\langle\widetilde{a}_{n}, \widetilde{b}_{n}\right\rangle\right) \\
& =f\left(\left\langle\widetilde{a}_{1}^{\prime}, \widetilde{b}_{1}^{\prime}\right\rangle,\left\langle\tilde{a}_{2}^{\prime}, \tilde{b}_{2}^{\prime}\right\rangle, \ldots,\left\langle\widetilde{a}_{n}^{\prime}, \widetilde{b}_{n}^{\prime}\right\rangle\right) .
\end{aligned}
$$

Theorem 13 (commutativity-distance measure). Assume that $f$ is the ITOWD operator; then

$$
\begin{aligned}
f & \left(\left\langle\widetilde{a}_{1}, \widetilde{b}_{1}\right\rangle,\left\langle\widetilde{a}_{2}, \widetilde{b}_{2}\right\rangle, \ldots,\left\langle\widetilde{a}_{n}, \widetilde{b}_{n}\right\rangle\right) \\
& =f\left(\left\langle\widetilde{b}_{1}, \tilde{a}_{1}\right\rangle,\left\langle\widetilde{b}_{2}, \tilde{a}_{2}\right\rangle, \ldots,\left\langle\widetilde{b}_{n}, \tilde{a}_{n}\right\rangle\right) .
\end{aligned}
$$

Theorem 14 (monotonicity). Assume that $f$ is the ITOWD operator. If $d_{I T D}\left(\widetilde{a}_{i}, \widetilde{b}_{i}\right) \geq d_{I T D}\left(\tilde{a}_{i}^{\prime}, \widetilde{b}_{i}^{\prime}\right)$, for all $i$, then

$$
\begin{aligned}
& f\left(\left\langle\widetilde{a}_{1}, \tilde{b}_{1}\right\rangle,\left\langle\tilde{a}_{2}, \widetilde{b}_{2}\right\rangle, \ldots,\left\langle\tilde{a}_{n}, \widetilde{b}_{n}\right\rangle\right) \\
& \quad \geq f\left(\left\langle\tilde{a}_{1}^{\prime}, \tilde{b}_{1}^{\prime}\right\rangle,\left\langle\tilde{a}_{2}^{\prime}, \tilde{b}_{2}^{\prime}\right\rangle, \ldots,\left\langle\tilde{a}_{n}^{\prime}, \tilde{b}_{n}^{\prime}\right\rangle\right) .
\end{aligned}
$$

Theorem 15 (idempotency). Assume that $f$ is the ITOWD operator. If $d_{I T D}\left(\widetilde{a}_{i}, \widetilde{b}_{i}\right)=d$, for all $i$, then

$$
f\left(\left\langle\widetilde{a}_{1}, \widetilde{b}_{1}\right\rangle,\left\langle\widetilde{a}_{2}, \widetilde{b}_{2}\right\rangle, \ldots,\left\langle\widetilde{a}_{n}, \widetilde{b}_{n}\right\rangle\right)=d .
$$

The proofs of the above theorems are straightforward and thus are omitted.

Theorem 16 (bounded). Assume that $f$ is the ITOWD operator; then

$$
\begin{aligned}
& \min \left\{d_{\text {ITD }}\left(\tilde{a}_{i}, \widetilde{b}_{i}\right)\right\} \\
& \quad \leq f\left(\left\langle\widetilde{a}_{1}, \widetilde{b}_{1}\right\rangle,\left\langle\widetilde{a}_{2}, \widetilde{b}_{2}\right\rangle, \ldots,\left\langle\widetilde{a}_{n}, \widetilde{b}_{n}\right\rangle\right) \\
& \quad \leq \max \left\{d_{I T D}\left(\widetilde{a}_{i}, \widetilde{b}_{i}\right)\right\} .
\end{aligned}
$$

Proof. Let $\max \left\{d_{\mathrm{ITD}}\left(\widetilde{a}_{i}, \widetilde{b}_{i}\right)\right\}=t$, and let $\min \left\{d_{\mathrm{ITD}}\left(\widetilde{a}_{i}, \widetilde{b}_{i}\right)\right\}=r$; then

$$
\begin{aligned}
f & \left(\left\langle\widetilde{a}_{1}, \widetilde{b}_{1}\right\rangle,\left\langle\widetilde{a}_{2}, \widetilde{b}_{2}\right\rangle, \ldots,\left\langle\widetilde{a}_{n}, \widetilde{b}_{n}\right\rangle\right) \\
& =\sum_{j=1}^{n} \omega_{j} d_{\text {ITD }}\left(\widetilde{a}_{\sigma(j)}, \widetilde{b}_{\sigma(j)}\right) \leq \sum_{j=1}^{n} \omega_{j} t=t \sum_{j=1}^{n} \omega_{j}=t, \\
f & \left(\left\langle\widetilde{a}_{1}, \widetilde{b}_{1}\right\rangle,\left\langle\widetilde{a}_{2}, \widetilde{b}_{2}\right\rangle, \ldots,\left\langle\widetilde{a}_{n}, \widetilde{b}_{n}\right\rangle\right) \\
& =\sum_{j=1}^{n} \omega_{j} d_{\text {ITD }}\left(\tilde{a}_{\sigma(j)}, \widetilde{b}_{\sigma(j)}\right) \geq \sum_{j=1}^{n} \omega_{j} r=r \sum_{j=1}^{n} \omega_{j}=r .
\end{aligned}
$$

Therefore,

$$
\begin{aligned}
& \min \left\{d_{\mathrm{ITD}}\left(\widetilde{a}_{i}, \widetilde{b}_{i}\right)\right\} \\
& \quad \leq f\left(\left\langle\widetilde{a}_{1}, \widetilde{b}_{1}\right\rangle,\left\langle\widetilde{a}_{2}, \widetilde{b}_{2}\right\rangle, \ldots,\left\langle\widetilde{a}_{n}, \widetilde{b}_{n}\right\rangle\right) \\
& \quad \leq \max \left\{d_{\mathrm{ITD}}\left(\widetilde{a}_{i}, \widetilde{b}_{i}\right)\right\} .
\end{aligned}
$$

Another important issue is the determination of the weighting vector associated with the ITOWD operator. In the literature, various methods have been suggested for the OWA weights generation, which can also be implemented for the ITOWD operator, such as the normal distribution based method [42], the maximum Bayesian entropy method [43], and the least squares based method [44]. Inspired by [8, 45], in the following, we give three ways to determine the ITOWD weights.

(1) Let

$$
\omega_{j}=\frac{d_{\mathrm{ITD}}\left(\tilde{a}_{\sigma(j)}, \widetilde{b}_{\sigma(j)}\right)}{\sum_{j=1}^{1} d_{\mathrm{ITD}}\left(\widetilde{a}_{\sigma(j)}, \widetilde{b}_{\sigma(j)}\right)}, \quad j=1,2, \ldots, n ;
$$

then $\omega_{j+1} \geq \omega_{j} \geq 0, j=1,2, \ldots, n-1$, and $\sum_{j=1}^{1} \omega_{j}=$ 1 .

(2) Let

$$
\omega_{j}=\frac{e^{-d_{\mathrm{ITD}}\left(\tilde{a}_{\sigma(j)}, \tilde{b}_{\sigma(j)}\right)}}{\sum_{j=1}^{1} e^{-d_{\mathrm{ITD}}\left(\tilde{a}_{\sigma(j)}, \tilde{b}_{\sigma(j)}\right)}}, \quad j=1,2, \ldots, n ;
$$

then $0 \leq \omega_{j+1} \leq \omega_{j}, j=1,2, \ldots, n-1$, and $\sum_{j=1}^{1} \omega_{j}=$ 1.

(3) Let

$$
\begin{aligned}
& \dot{d}_{\mathrm{ITD}}\left(\tilde{a}_{\sigma(j)}, \widetilde{b}_{\sigma(j)}\right)=\frac{1}{n} \sum_{j=1}^{1} d_{\mathrm{ITD}}\left(\tilde{a}_{\sigma(j)}, \widetilde{b}_{\sigma(j)}\right), \\
& \ddot{d}\left(d_{\mathrm{ITD}}\left(\widetilde{a}_{\sigma(j)}, \widetilde{b}_{\sigma(j)}\right), \dot{d}_{\mathrm{ITD}}\left(\widetilde{a}_{\sigma(j)}, \widetilde{b}_{\sigma(j)}\right)\right) \\
& =\left|d_{\mathrm{ITD}}\left(\widetilde{a}_{\sigma(j)}, \widetilde{b}_{\sigma(j)}\right)-\dot{d}_{\mathrm{ITD}}\left(\widetilde{a}_{\sigma(j)}, \widetilde{b}_{\sigma(j)}\right)\right| ;
\end{aligned}
$$


then we define

$\omega_{j}$

$$
\begin{array}{r}
=\frac{1-\ddot{d}\left(d_{\mathrm{ITD}}\left(\widetilde{a}_{\sigma(j)}, \widetilde{b}_{\sigma(j)}\right), \dot{d}_{\mathrm{ITD}}\left(\widetilde{a}_{\sigma(j)}, \widetilde{b}_{\sigma(j)}\right)\right)}{\sum_{j=1}^{1}\left(1-\ddot{d}\left(d_{\mathrm{ITD}}\left(\widetilde{a}_{\sigma(j)}, \widetilde{b}_{\sigma(j)}\right), \dot{d}_{\mathrm{ITD}}\left(\widetilde{a}_{\sigma(j)}, \widetilde{b}_{\sigma(j)}\right)\right)\right)}, \\
j=1,2, \ldots, n,
\end{array}
$$

from which we get $\omega_{j} \geq 0, j=1,2, \ldots, n$, and $\sum_{j=1}^{1} \omega_{j}=1$.

Note that the weight vector derived from (22) is a monotonic decreasing sequence, the weight vector derived from (23) is a monotonic increasing sequence, and the weight vector derived from (25) combines the above two cases; that is, the closer the value $d_{\mathrm{ITD}}\left(\widetilde{a}_{\sigma(j)}, \widetilde{b}_{\sigma(j)}\right)$ to the mean $\dot{d}_{\text {ITD }}\left(\tilde{a}_{\sigma(j)}, \widetilde{b}_{\sigma(j)}\right)$, the larger the weight $\omega_{j}$.

Clearly, the fundamental characteristic of the ITWD operator is that it considers the importance of each given interval 2-tuple distance, whereas the fundamental characteristic of the ITOWD operator is the reordering step, and it weights all the ordered positions of the interval 2-tuple distances instead of weighting the given interval 2-tuple distances themselves. Motived by the idea of the linguistic hybrid geometric averaging (LHGA) operator [42], in the following, we develop an interval 2-tuple hybrid weighted distance (ITHWD) operator that weights both the given interval 2-tuple distances and their ordered positions.

Definition 17. An ITHWD operator of dimension $n$ is a mapping ITHWD: $\widetilde{S}^{n} \times \widetilde{S}^{n} \rightarrow \widehat{S}$, which has an associated weight vector $\omega=\left(\omega_{1}, \omega_{2}, \ldots, \omega_{n}\right)^{T}$, with $\omega_{j} \in[0,1]$ and $\sum_{j=1}^{n} \omega_{j}=1$, such that

$$
\begin{aligned}
& \operatorname{ITHWD}\left(\left\langle\widetilde{a}_{1}, \widetilde{b}_{1}\right\rangle,\left\langle\widetilde{a}_{2}, \widetilde{b}_{2}\right\rangle, \ldots,\left\langle\widetilde{a}_{n}, \widetilde{b}_{n}\right\rangle\right) \\
& =\sum_{j=1}^{n} \omega_{j} d_{\operatorname{ITD}}\left(\dot{\tilde{a}}_{\sigma(j)}, \dot{\tilde{b}}_{\sigma(j)}\right),
\end{aligned}
$$

where $d_{\text {ITD }}\left(\dot{\tilde{a}}_{\sigma(j)}, \dot{\widetilde{b}}_{\sigma(j)}\right)$ is the $j$ th largest of the weighted interval 2-tuple distance $d_{\text {ITD }}\left(\dot{\tilde{a}}_{i}, \dot{\widetilde{b}}_{i}\right)\left(d_{\text {ITD }}\left(\dot{\tilde{a}}_{i}, \dot{\widetilde{b}}_{i}\right)=n w_{i} d_{\text {ITD }}\left(\widetilde{a}_{i}\right.\right.$, $\left.\left.\widetilde{b}_{i}\right), i=1,2, \ldots, n\right), w=\left(w_{1}, w_{2}, \ldots, w_{n}\right)^{T}$ is the weight vector of $d_{\mathrm{ITD}}\left(\widetilde{a}_{i}, \widetilde{b}_{i}\right)(i=1,2, \ldots, n)$, with $w_{i} \in[0,1]$ and $\sum_{i=1}^{n} w_{i}=1$, and $n$ is the balancing coefficient.

In particular, if $w=(1 / n, 1 / n, \ldots, 1 / n)^{T}$, then the ITHWD is reduced to the ITOWD operator; if $\omega=(1 / n, 1 / n$, $\ldots, 1 / n)^{T}$, then the ITHWD is reduced to the ITWD operator. Moreover, if $\widetilde{A}$ and $\widetilde{B}$ are degenerated to $\widehat{A}$ and $\widehat{B}$, then the ITHWD is reduced to the 2-tuple hybrid weighted distance (THWD) operator:

$$
\begin{aligned}
& \operatorname{THWD}\left(\left\langle\widehat{a}_{1}, \widehat{b}_{1}\right\rangle,\left\langle\widehat{a}_{2}, \widehat{b}_{2}\right\rangle, \ldots,\left\langle\widehat{a}_{n}, \widehat{b}_{n}\right\rangle\right) \\
& =\sum_{j=1}^{n} \omega_{j} d_{\mathrm{TD}}\left(\dot{\hat{a}}_{\sigma(j)}, \dot{\hat{b}}_{\sigma(j)}\right),
\end{aligned}
$$

where $d_{\mathrm{TD}}\left(\dot{\hat{a}}_{\sigma(j)}, \dot{\hat{b}}_{\sigma(j)}\right)$ is the jth largest of the weighted 2tuple distance $d_{\mathrm{TD}}\left(\dot{\widehat{a}}_{j}, \dot{\widehat{b}}_{j}\right)\left(d_{\mathrm{TD}}\left(\dot{\widehat{a}}_{j}, \dot{\hat{b}}_{j}\right)=n w_{i} d_{\mathrm{TD}}\left(\widehat{a}_{i}, \widehat{b}_{i}\right), i=\right.$ $1,2, \ldots, n), w=\left(w_{1}, w_{2}, \ldots, w_{n}\right)^{T}$ is the weight vector of $d_{\mathrm{TD}}\left(\dot{\hat{a}}_{j}, \dot{\hat{b}}_{j}\right)(i=1,2, \ldots, n)$, with $w_{i} \in[0,1]$ and $\sum_{i=1}^{n} w_{i}=1$, and $n$ is the balancing coefficient.

3.2. Generalizations of the ITOWD Operator. In what follows, generalizations of the ITOWD operator are presented by using the generalized and the quasi-arithmetic means.

Definition 18. A generalized interval 2-tuple ordered weighted distance (GITOWD) operator of dimension $n$ is a mapping GITOWD: $\widetilde{S}^{n} \times \widetilde{S}^{n} \rightarrow \widehat{S}$, which has an associated weight vector $\omega=\left(\omega_{1}, \omega_{2}, \ldots, \omega_{n}\right)^{T}$, with $\omega_{j} \in[0,1]$ and $\sum_{j=1}^{n} \omega_{j}=1$, such that

$$
\begin{aligned}
& \operatorname{GITOWD}\left(\left\langle\tilde{a}_{1}, \tilde{b}_{1}\right\rangle,\left\langle\tilde{a}_{2}, \tilde{b}_{2}\right\rangle, \ldots,\left\langle\tilde{a}_{n}, \tilde{b}_{n}\right\rangle\right) \\
& =\left(\sum_{j=1}^{n} \omega_{j} d_{\mathrm{ITD}}^{\lambda}\left(\tilde{a}_{\sigma(j)}, \tilde{b}_{\sigma(j)}\right)\right)^{1 / \lambda},
\end{aligned}
$$

where $d_{\text {ITD }}\left(\widetilde{a}_{\sigma(j)}, \widetilde{b}_{\sigma(j)}\right)$ is the $j$ th largest of the interval 2tuple distance $d_{\mathrm{ITD}}\left(\widetilde{a}_{i}, \widetilde{b}_{i}\right)$ and $\lambda$ is a parameter such that $\lambda \epsilon$ $(-\infty,+\infty)-\{0\}$.

Similar to the OWA and the GOWA operators [1, 46], the GITOWD operator has many desirable properties, such as commutativity, monotonicity, boundedness, and idempotency. Particularly, if there are ties between interval 2-tuple distances, as in the case of the ITOWD operator, we replace each of the tied arguments by their generalized mean in the process of aggregation. If $\widetilde{A}$ and $\widetilde{B}$ are degenerated to $\widehat{A}$ and $\widehat{B}$, then we can get the generalized 2-tuple ordered weighted distance (GTOWD) operator. The GITOWD operator provides a parameterized family of aggregation operators. In order to study this family, we can analyze the weighting vector $\omega$ or the parameter $\lambda$. By choosing a different manifestation of the weighting vector in the GITOWD operator, we are able to obtain different types of distance operators:

(i) The interval 2-tuple maximum distance is found if $\omega_{1}=1$ and $\omega_{j}=0$, for all $j \neq 1$.

(ii) The interval 2-tuple minimum distance is found if $\omega_{n}=1$ and $\omega_{j}=0$, for all $j \neq n$.

(iii) The generalized interval 2-tuple normalized distance (GITND) operator is formed when $\omega_{j}=1 / n$, for all $j$.

(iv) The generalized interval 2-tuple weighted distance (GITWD) operator is obtained when the position of $d_{\mathrm{ITD}}\left(\widetilde{a}_{i}, \widetilde{b}_{i}\right)$ is the same as the ordered position of $d_{\text {ITD }}\left(\tilde{a}_{\sigma(j)}, \tilde{b}_{\sigma(j)}\right)$.

Some special cases can also be obtained with the change of the parameter $\lambda$ :

(i) If $\lambda=1$, then the GITOWD is reduced to the ITOWD operator. 
(ii) If $\lambda \rightarrow 0$, then the GITOWD is reduced to the interval 2-tuple ordered weighted geometric distance (ITOWGD) operator.

(iii) If $\lambda=-1$, then the GITOWD is reduced to the interval 2-tuple ordered weighted harmonic distance (ITOWHD) operator.

(iv) If $\lambda=2$, then the GITOWD is reduced to the interval 2-tuple ordered weighted Euclidean distance (ITOWED) operator.

(v) If $\lambda=3$, then the GITOWD is reduced to the interval 2-tuple ordered weighted cubic distance (ITOWCD) operator.

Definition 19. A quasi-arithmetic interval 2-tuple ordered weighted distance (Quasi-ITOWD) operator of dimension $n$ is a mapping Quasi-ITOWD: $\widetilde{S}^{n} \times \widetilde{S}^{n} \rightarrow \widehat{S}$, which has an associated weight vector $\omega=\left(\omega_{1}, \omega_{2}, \ldots, \omega_{n}\right)^{T}$, with $\omega_{j} \in$ $[0,1]$ and $\sum_{j=1}^{n} \omega_{j}=1$, such that

$$
\begin{aligned}
& \operatorname{Quasi-ITOWD}\left(\left\langle\tilde{a}_{1}, \tilde{b}_{1}\right\rangle,\left\langle\tilde{a}_{2}, \tilde{b}_{2}\right\rangle, \ldots,\left\langle\tilde{a}_{n}, \tilde{b}_{n}\right\rangle\right) \\
& =g^{-1}\left(\sum_{j=1}^{n} \omega_{j} g\left(d_{\operatorname{ITD}}\left(\tilde{a}_{\sigma(j)}, \tilde{b}_{\sigma(j)}\right)\right)\right),
\end{aligned}
$$

where $d_{\text {ITD }}\left(\widetilde{a}_{\sigma(j)}, \widetilde{b}_{\sigma(j)}\right)$ is the $j$ th largest of the interval 2-tuple distance $d_{\mathrm{ITD}}\left(\widetilde{a}_{i}, \tilde{b}_{i}\right)$ and $g$ is a general continuous strictly monotone function.

As we can see, the GITOWD operator is a particular case of the Quasi-ITOWD operator when $g(x)=x^{\lambda}$. If $\widetilde{A}$ and $\widetilde{B}$ are degenerated to $\widehat{A}$ and $\widehat{B}$, then we can get the quasi-arithmetic 2-tuple ordered weighted distance (QuasiTOWD) operator. Note that all properties and particular cases commented in the GITOWD operator can also be discussed in this generalization.

\section{The Proposed Multicriteria Group Supplier Selection Method}

In this section, we develop an approach based on the proposed interval 2-tuple linguistic distance operators for solving multicriteria group supplier selection problems.

Suppose that a group supplier selection problem has $l$ decision makers $\mathrm{DM}_{k}(k=1,2, \ldots, l), m$ alternatives $A_{i}(i=$ $1,2, \ldots, m)$, and $n$ decision criteria $C_{j}(j=1,2, \ldots, n)$. Each decision maker $\mathrm{DM}_{k}$ is given a weight $v_{k}>0(k=1,2, \ldots, l)$ satisfying $\sum_{k=1}^{l} v_{k}=1$ to reflect his/her relative importance in the supplier selection process. Let $D_{k}=\left(d_{i j}^{k}\right)_{m \times n}$ be the linguistic decision matrix of the $k$ th decision maker, where $d_{i j}^{k}$ is the linguistic information provided by $\mathrm{DM}_{k}$ on the assessment of $A_{i}$ with respect to $C_{j}$. In addition, decision makers may use different linguistic term sets to express their assessment values.

Next, we apply the ITWA and the GITOWD operators for multicriteria group supplier selection under interval 2-tuple linguistic environment.
Step 1. Convert the linguistic decision matrix $D_{k}=\left(d_{i j}^{k}\right)_{m \times n}$ into the interval 2-tuple linguistic decision matrix $\widetilde{R}_{k}=$ $\left(\widetilde{r}_{i j}^{k}\right)_{m \times n}=\left(\left[\left(r_{i j}^{k}, 0\right),\left(t_{i j}^{k}, 0\right)\right]\right)_{m \times n}$, where $r_{i j}^{k}, t_{i j}^{k} \in S, S=\left\{s_{0}, s_{1}\right.$, $\left.\ldots, s_{g}\right\}$ and $r_{i j}^{k} \leq t_{i j}^{k}$.

Suppose that $\mathrm{DM}_{k}$ provides his assessments in a set of five linguistic terms $S: S=\left\{s_{0}=\right.$ very poor, $s_{1}=$ poor, $s_{2}=$ medium, $s_{3}=$ good, $s_{4}=$ very good $\}$. The linguistic information provided in the decision matrix $D_{k}$ can be converted into corresponding interval 2-tuple linguistic assessments according to the following ways:

(i) A certain grade such as poor, which can be written as $\left[\left(s_{1}, 0\right),\left(s_{1}, 0\right)\right]$

(ii) An interval such as poor-medium, which means that the assessment of an alternative concerning the criterion under consideration is between poor and medium. This can be expressed as $\left[\left(s_{1}, 0\right),\left(s_{2}, 0\right)\right]$.

Remark 20. In particular supplier selection problems, there exist many situations where information may be unquantifiable due to its nature, or the precise quantitative information may be unavailable or the cost for its computation is too high. Thus, it is more reasonable and natural for decision makers to make their judgments by using linguistic expressions. Generally, three main methods have been introduced for dealing with qualitative assessments [31, 39, 47]. The first method is based on membership functions [48], which converts linguistic information into fuzzy numbers by means of a membership function. However, this method led to a certain degree of information loss in the transformation process. The second method is based on linguistic symbols [49] that made computations on the subscripts of linguistic terms and was easy to operate. However, this approach may lead to inflexibility for different semantics. The third method is based on linguistic 2-tuples [29], which can avoid the information distortion and loss in linguistic information processing and has been widely utilized for managing linguistic MCDM problems. Therefore, to deal with linguistic information more reasonably and accurately, the decision maskers' assessments on alternative suppliers are first transformed into interval 2-tuples in the proposed approach.

Step 2. Utilize the ITWA operator

$$
\begin{array}{r}
\tilde{r}_{i j}=\left[\left(r_{i j}, \alpha_{i j}\right),\left(t_{i j}, \varepsilon_{i j}\right)\right]=\operatorname{ITWA}\left(\widetilde{r}_{i j}^{1}, \widetilde{r}_{i j}^{2}, \ldots, \widetilde{r}_{i j}^{l}\right) \\
=\Delta\left[\sum_{k=1}^{l} v_{k} \Delta^{-1}\left(r_{i j}^{k}, 0\right), \sum_{k=1}^{l} v_{k} \Delta^{-1}\left(t_{i j}^{k}, 0\right)\right], \\
i=1,2, \ldots, m, j=1,2, \ldots, n,
\end{array}
$$

to aggregate all the interval 2-tuple linguistic decision matrices $\widetilde{R}_{k}(k=1,2, \ldots, l)$ into a collective interval 2-tuple linguistic decision matrix $\widetilde{R}=\left(\widetilde{r}_{i j}\right)_{m \times n}$. 
Step 3. Determine the ideal level of each criterion in order to characterize the collective ideal alternative $\tilde{r}^{*}=\left(\widetilde{r}_{1}^{*}, \widetilde{r}_{2}^{*}\right.$, $\left.\ldots, \widetilde{r}_{n}^{*}\right)$, where

$$
\tilde{r}_{j}^{*}=\left[\left(r_{j}^{*}, \alpha_{j}^{*}\right),\left(t_{j}^{*}, \varepsilon_{j}^{*}\right)\right], \quad j=1,2, \ldots, n .
$$

Step 4. Calculate the separation measure $S_{i}^{+}$of each alternative from the ideal alternative by using the GITOWD operator:

$$
\begin{aligned}
S_{i}^{+} & =\operatorname{GiTOWD}\left(\left\langle\widetilde{r}_{i 1}, \widetilde{r}_{1}^{*}\right\rangle,\left\langle\widetilde{r}_{i 2}, \widetilde{r}_{2}^{*}\right\rangle, \ldots,\left\langle\widetilde{r}_{i j}, \widetilde{r}_{j}^{*}\right\rangle\right) \\
& =\left(\sum_{j=1}^{n} \omega_{j} d_{\mathrm{ITD}}^{\lambda}\left(\widetilde{r}_{i \sigma(j)}, \widetilde{r}_{\sigma(j)}^{*}\right)\right)^{1 / \lambda}, \quad i=1,2, \ldots, m,
\end{aligned}
$$

where $d_{\mathrm{ITD}}^{\lambda}\left(\tilde{r}_{i \sigma(j)}, \tilde{r}_{\sigma(j)}^{*}\right)$ is the $j$ th largest of the interval 2-tuple distance $d_{\text {ITD }}\left(\widetilde{r}_{i j}, \widetilde{r}_{j}^{*}\right) ; \omega=\left(\omega_{1}, \omega_{2}, \ldots, \omega_{n}\right)^{T}$ is the weighting vector of the GITOWD operator such that $\omega_{j} \in[0,1]$ and $\sum_{j=1}^{n} \omega_{j}=1$. Note that it is possible to consider a wide range of GITOWD operators such as those described in Section 3.

Step 5. Rank all the alternatives $A_{i}(i=1,2, \ldots, m)$ and select the best one(s) according to the increasing order of their separation measures.

Step 6. End.

\section{An Illustrative Example}

5.1. Example Illustration. In this section, we develop an illustrative example of the new approach in a group decision making problem of supplier selection. Suppose that a tertiary care hospital desires to select the most appropriate supplier for one of the key medical devices in the general anesthesia process. After preliminary screening, six suppliers, $A_{i}(i=1,2, \ldots, 6)$, have remained as alternatives for further evaluation. In order to evaluate the alternative suppliers and select the best one, an expert committee of three decision makers, $\mathrm{DM}_{1}, \mathrm{DM}_{2}$ and $\mathrm{DM}_{3}$, has been formed. The selection decision is made on the basis of one objective and five criteria $C_{j}(j=1,2, \ldots, 5)$. These criteria, which are critical for the supplier selection, are defined as follows:

$$
\begin{aligned}
& C_{1}: \text { technical capability } \\
& C_{2}: \text { delivery performance } \\
& C_{3}: \text { product quality } \\
& C_{4}: \text { flexibility } \\
& C_{5}: \text { price/cost }
\end{aligned}
$$

The three decision makers employ different linguistic term sets to assess the suitability of the suppliers with respect to the above selection criteria. Specifically, $\mathrm{DM}_{1}$ provides his assessments by using the linguistic term set $A ; \mathrm{DM}_{2}$ provides his assessments using $B ; \mathrm{DM}_{3}$ provides her assessments using $C$. These linguistic term sets are denoted as follows:

$$
\begin{aligned}
A= & \left\{a_{0}=\operatorname{very} \operatorname{poor}(\mathrm{VP}), a_{1}=\operatorname{poor}(\mathrm{P}), a_{2}\right. \\
& =\operatorname{medium}(\mathrm{M}), a_{3}=\operatorname{good}(\mathrm{G}), a_{4} \\
& =\operatorname{very} \operatorname{good}(\mathrm{VG})\}, \\
B & =\left\{b_{0}=\operatorname{very} \operatorname{poor}(\mathrm{VP}), b_{1}=\operatorname{poor}(\mathrm{P}), b_{2}\right. \\
& =\operatorname{medium} \operatorname{poor}(\mathrm{MP}), b_{3}=\operatorname{medium}(\mathrm{M}), b_{4} \\
& =\operatorname{medium} \operatorname{good}(\mathrm{MG}), b_{5}=\operatorname{good}(\mathrm{G}), b_{6} \\
& =\operatorname{very} \operatorname{good}(\mathrm{VG})\}, \\
C & =\left\{c_{0}=\operatorname{extra} \operatorname{poor}(\mathrm{EP}), c_{1}=\operatorname{very} \operatorname{poor}(\mathrm{VP}), c_{2}\right. \\
& =\operatorname{poor}(\mathrm{P}), c_{3}=\operatorname{medium} \operatorname{poor}(\mathrm{MP}), c_{4} \\
& =\operatorname{medium}(\mathrm{M}), c_{5}=\operatorname{medium} \operatorname{good}(\mathrm{MG}), c_{6} \\
& =\operatorname{good}(\mathrm{G}), c_{7}=\operatorname{very} \operatorname{good}(\operatorname{VG}), c_{8} \\
& =\operatorname{extra} \operatorname{good}(\mathrm{EG})\} .
\end{aligned}
$$

The linguistic assessments of the six alternatives on each criterion provided by the three decision makers are presented in Table 1.

With this information, we can make an aggregation in order to make a decision. First, we convert the linguistic decision matrix shown in Table 1 into the interval 2-tuple linguistic decision matrix $\widetilde{R}_{k}=\left(\left[\left(r_{i j}^{k}, 0\right),\left(t_{i j}^{k}, 0\right)\right]\right)_{6 \times 5}$, which is depicted in Table 2 . Then, we aggregate the information of the three experts to obtain a collective interval 2-tuple linguistic decision matrix. We use the ITWA operator to obtain this matrix while assuming that $v=(0.3,0.4,0.3)^{T}$. The results are shown in Table 3.

According to their objectives, the group of experts establishes the collective ideal supplier shown in Table 4 .

It is now possible to develop different methods based on the GITOWD operator for the selection of the optimum supplier. In this example, we consider the interval 2-tuple maximum distance, the interval 2-tuple minimum distance, the ITND, the ITWD, the ITHWD, the ITOWD, the ITOWGD, the ITOWHD, the ITOWED, and the ITOWCD operators. For convenience, we assume the following weighting vector: $\omega=(0.112,0.236,0.304,0.236,0.112)^{T}$, which is derived by the normal distribution based method [42]. The aggregated results are presented in Tables 5 and 6 and the rankings of the alternative suppliers for each particular case are shown in Table 7.

As we can see, depending on the distance operator used, the ranking orders of the six suppliers are different. Due to the fact that each particular type of the GITOWD operator may lead to different results, the decision maker can select for his decision the one that is in closest accordance with his interests. However, in this example, it is clear that the best choice is $A_{2}$, although in some exceptional situations the alternative suppliers such as $A_{1}, A_{4}$, or $A_{5}$ could be optimal. 
TABLE 1: Linguistic assessments of the suppliers.

\begin{tabular}{|c|c|c|c|c|c|c|}
\hline \multirow{2}{*}{ Decision makers } & \multirow{2}{*}{ Alternatives } & \multicolumn{5}{|c|}{ Criteria } \\
\hline & & $C_{1}$ & $\mathrm{C}_{2}$ & $C_{3}$ & $C_{4}$ & $C_{5}$ \\
\hline \multirow{6}{*}{$\mathrm{DM}_{1}$} & $A_{1}$ & G-VG & M-G & G & $\mathrm{M}$ & G \\
\hline & $A_{2}$ & VG & G & VG & $M-G$ & G \\
\hline & $A_{3}$ & M & M & $\mathrm{P}-\mathrm{M}$ & G-VG & M \\
\hline & $A_{4}$ & G & G-VG & M & G & G-VG \\
\hline & $A_{5}$ & M & VG & G & VG & G \\
\hline & $A_{6}$ & G & M-G & VG & G & M-G \\
\hline \multirow{6}{*}{$\mathrm{DM}_{2}$} & $A_{1}$ & VG & M & G-VG & M & G \\
\hline & $A_{2}$ & MG & VG & G & MG & MG \\
\hline & $A_{3}$ & $M-G$ & MG & M & MG & G \\
\hline & $A_{4}$ & G & VG & G & MG-G & M \\
\hline & $A_{5}$ & $M-G$ & M & G & VG & VG \\
\hline & $A_{6}$ & MG & M-G & G & G & VG \\
\hline \multirow{6}{*}{$\mathrm{DM}_{3}$} & $A_{1}$ & M-MG & G & MG & G & M \\
\hline & $A_{2}$ & VG & VG & MG & VG & G \\
\hline & $A_{3}$ & VG & M & G & MG & VG \\
\hline & $A_{4}$ & EG & VG & G & G & VG \\
\hline & $A_{5}$ & G & MG & G-VG & G & MG \\
\hline & $A_{6}$ & M & M-G & G & G & MG \\
\hline
\end{tabular}

TABLE 2: Interval 2-tuple linguistic decision matrix.

\begin{tabular}{|c|c|c|c|c|c|c|}
\hline \multirow{2}{*}{ Decision makers } & \multirow{2}{*}{ Alternatives } & \multicolumn{5}{|c|}{ Criteria } \\
\hline & & $C_{1}$ & $\mathrm{C}_{2}$ & $\mathrm{C}_{3}$ & $\mathrm{C}_{4}$ & $C_{5}$ \\
\hline \multirow{6}{*}{$\mathrm{DM}_{1}$} & $A_{1}$ & {$\left[\left(a_{3}, 0\right),\left(a_{4}, 0\right)\right]$} & {$\left[\left(a_{2}, 0\right),\left(a_{3}, 0\right)\right]$} & {$\left[\left(a_{3}, 0\right),\left(a_{3}, 0\right)\right]$} & {$\left[\left(a_{2}, 0\right),\left(a_{2}, 0\right)\right]$} & {$\left[\left(a_{3}, 0\right),\left(a_{3}, 0\right)\right]$} \\
\hline & $A_{2}$ & {$\left[\left(a_{4}, 0\right),\left(a_{4}, 0\right)\right]$} & {$\left[\left(a_{3}, 0\right),\left(a_{3}, 0\right)\right]$} & {$\left[\left(a_{4}, 0\right),\left(a_{4}, 0\right)\right]$} & {$\left[\left(a_{2}, 0\right),\left(a_{3}, 0\right)\right]$} & {$\left[\left(a_{3}, 0\right),\left(a_{3}, 0\right)\right]$} \\
\hline & $A_{3}$ & {$\left[\left(a_{2}, 0\right),\left(a_{2}, 0\right)\right]$} & {$\left[\left(a_{2}, 0\right),\left(a_{2}, 0\right)\right]$} & {$\left[\left(a_{1}, 0\right),\left(a_{2}, 0\right)\right]$} & {$\left[\left(a_{3}, 0\right),\left(a_{4}, 0\right)\right]$} & {$\left[\left(a_{2}, 0\right),\left(a_{2}, 0\right)\right]$} \\
\hline & $A_{4}$ & {$\left[\left(a_{3}, 0\right),\left(a_{3}, 0\right)\right]$} & {$\left[\left(a_{3}, 0\right),\left(a_{4}, 0\right)\right]$} & {$\left[\left(a_{2}, 0\right),\left(a_{2}, 0\right)\right]$} & {$\left[\left(a_{3}, 0\right),\left(a_{3}, 0\right)\right]$} & {$\left[\left(a_{3}, 0\right),\left(a_{4}, 0\right)\right]$} \\
\hline & $A_{5}$ & {$\left[\left(a_{2}, 0\right),\left(a_{2}, 0\right)\right]$} & {$\left[\left(a_{4}, 0\right),\left(a_{4}, 0\right)\right]$} & {$\left[\left(a_{3}, 0\right),\left(a_{3}, 0\right)\right]$} & {$\left[\left(a_{4}, 0\right),\left(a_{4}, 0\right)\right]$} & {$\left[\left(a_{3}, 0\right),\left(a_{3}, 0\right)\right]$} \\
\hline & $A_{6}$ & {$\left[\left(a_{3}, 0\right),\left(a_{3}, 0\right)\right]$} & {$\left[\left(a_{2}, 0\right),\left(a_{3}, 0\right)\right]$} & {$\left[\left(a_{4}, 0\right),\left(a_{4}, 0\right)\right]$} & {$\left[\left(a_{3}, 0\right),\left(a_{3}, 0\right)\right]$} & {$\left[\left(a_{2}, 0\right),\left(a_{3}, 0\right)\right]$} \\
\hline \multirow{6}{*}{$\mathrm{DM}_{2}$} & $A_{1}$ & {$\left[\left(b_{6}, 0\right),\left(b_{6}, 0\right)\right]$} & {$\left[\left(b_{3}, 0\right),\left(b_{3}, 0\right)\right]$} & {$\left[\left(b_{5}, 0\right),\left(b_{6}, 0\right)\right]$} & {$\left[\left(b_{3}, 0\right),\left(b_{3}, 0\right)\right]$} & {$\left[\left(b_{5}, 0\right),\left(b_{5}, 0\right)\right]$} \\
\hline & $A_{2}$ & {$\left[\left(b_{5}, 0\right),\left(b_{5}, 0\right)\right]$} & {$\left[\left(b_{6}, 0\right),\left(b_{6}, 0\right)\right]$} & {$\left[\left(b_{5}, 0\right),\left(b_{5}, 0\right)\right]$} & {$\left[\left(b_{4}, 0\right),\left(b_{4}, 0\right)\right]$} & {$\left[\left(b_{4}, 0\right),\left(b_{4}, 0\right)\right]$} \\
\hline & $A_{3}$ & {$\left[\left(b_{3}, 0\right),\left(b_{5}, 0\right)\right]$} & {$\left[\left(b_{4}, 0\right),\left(b_{4}, 0\right)\right]$} & {$\left[\left(b_{3}, 0\right),\left(b_{3}, 0\right)\right]$} & {$\left[\left(b_{4}, 0\right),\left(b_{4}, 0\right)\right]$} & {$\left[\left(b_{5}, 0\right),\left(b_{5}, 0\right)\right]$} \\
\hline & $A_{4}$ & {$\left[\left(b_{5}, 0\right),\left(b_{5}, 0\right)\right]$} & {$\left[\left(b_{6}, 0\right),\left(b_{6}, 0\right)\right]$} & {$\left[\left(b_{5}, 0\right),\left(b_{5}, 0\right)\right]$} & {$\left[\left(b_{4}, 0\right),\left(b_{5}, 0\right)\right]$} & {$\left[\left(b_{3}, 0\right),\left(b_{3}, 0\right)\right]$} \\
\hline & $A_{5}$ & {$\left[\left(b_{3}, 0\right),\left(b_{5}, 0\right)\right]$} & {$\left[\left(b_{3}, 0\right),\left(b_{3}, 0\right)\right]$} & {$\left[\left(b_{5}, 0\right),\left(b_{5}, 0\right)\right]$} & {$\left[\left(b_{6}, 0\right),\left(b_{6}, 0\right)\right]$} & {$\left[\left(b_{6}, 0\right),\left(b_{6}, 0\right)\right]$} \\
\hline & $A_{6}$ & {$\left[\left(b_{4}, 0\right),\left(b_{4}, 0\right)\right]$} & {$\left[\left(b_{3}, 0\right),\left(b_{5}, 0\right)\right]$} & {$\left[\left(b_{5}, 0\right),\left(b_{5}, 0\right)\right]$} & {$\left[\left(b_{5}, 0\right),\left(b_{5}, 0\right)\right]$} & {$\left[\left(b_{6}, 0\right),\left(b_{6}, 0\right)\right]$} \\
\hline \multirow{6}{*}{$\mathrm{DM}_{3}$} & $A_{1}$ & {$\left[\left(c_{4}, 0\right),\left(c_{5}, 0\right)\right]$} & {$\left[\left(c_{6}, 0\right),\left(c_{6}, 0\right)\right]$} & {$\left[\left(c_{5}, 0\right),\left(c_{5}, 0\right)\right]$} & {$\left[\left(c_{6}, 0\right),\left(c_{6}, 0\right)\right]$} & {$\left[\left(c_{4}, 0\right),\left(c_{4}, 0\right)\right]$} \\
\hline & $A_{2}$ & {$\left[\left(c_{7}, 0\right),\left(c_{7}, 0\right)\right]$} & {$\left[\left(c_{7}, 0\right),\left(c_{7}, 0\right)\right]$} & {$\left[\left(c_{5}, 0\right),\left(c_{5}, 0\right)\right]$} & {$\left[\left(c_{7}, 0\right),\left(c_{7}, 0\right)\right]$} & {$\left[\left(c_{6}, 0\right),\left(c_{6}, 0\right)\right]$} \\
\hline & $A_{3}$ & {$\left[\left(c_{7}, 0\right),\left(c_{7}, 0\right)\right]$} & {$\left[\left(c_{4}, 0\right),\left(c_{4}, 0\right)\right]$} & {$\left[\left(c_{6}, 0\right),\left(c_{6}, 0\right)\right]$} & {$\left[\left(c_{5}, 0\right),\left(c_{5}, 0\right)\right]$} & {$\left[\left(c_{7}, 0\right),\left(c_{7}, 0\right)\right]$} \\
\hline & $A_{4}$ & {$\left[\left(c_{8}, 0\right),\left(c_{8}, 0\right)\right]$} & {$\left[\left(c_{7}, 0\right),\left(c_{7}, 0\right)\right]$} & {$\left[\left(c_{6}, 0\right),\left(c_{6}, 0\right)\right]$} & {$\left[\left(c_{6}, 0\right),\left(c_{6}, 0\right)\right]$} & {$\left[\left(c_{7}, 0\right),\left(c_{7}, 0\right)\right]$} \\
\hline & $A_{5}$ & {$\left[\left(c_{6}, 0\right),\left(c_{6}, 0\right)\right]$} & {$\left[\left(c_{5}, 0\right),\left(c_{5}, 0\right)\right]$} & {$\left[\left(c_{6}, 0\right),\left(c_{7}, 0\right)\right]$} & {$\left[\left(c_{6}, 0\right),\left(c_{6}, 0\right)\right]$} & {$\left[\left(c_{5}, 0\right),\left(c_{5}, 0\right)\right]$} \\
\hline & $A_{6}$ & {$\left[\left(c_{4}, 0\right),\left(c_{4}, 0\right)\right]$} & {$\left[\left(c_{4}, 0\right),\left(c_{6}, 0\right)\right]$} & {$\left[\left(c_{6}, 0\right),\left(c_{6}, 0\right)\right]$} & {$\left[\left(c_{6}, 0\right),\left(c_{6}, 0\right)\right]$} & {$\left[\left(c_{5}, 0\right),\left(c_{5}, 0\right)\right]$} \\
\hline
\end{tabular}

TABLE 3: Collective interval 2-tuple linguistic decision matrix.

\begin{tabular}{lccccc}
\hline & $C_{1}$ & $C_{2}$ & $C_{3}$ & $C_{4}$ & $C_{5}$ \\
\hline$A_{1}$ & $\Delta[0.775,0.888]$ & $\Delta[0.575,0.650]$ & $\Delta[0.746,0.813]$ & $\Delta[0.575,0.575]$ & $\Delta[0.708,0.708]$ \\
$A_{2}$ & $\Delta[0.896,0.896]$ & $\Delta[0.888,0.888]$ & $\Delta[0.821,0.821]$ & $\Delta[0.679,0.754]$ & $\Delta[0.717,0.717]$ \\
$A_{3}$ & $\Delta[0.613,0.746]$ & $\Delta[0.567,0.567]$ & $\Delta[0.500,0.575]$ & $\Delta[0.679,0.754]$ & $\Delta[0.746,0.746]$ \\
$A_{4}$ & $\Delta[0.858,0.858]$ & $\Delta[0.888,0.963]$ & $\Delta[0.708,0.708]$ & $\Delta[0.717,0.783]$ & $\Delta[0.688,0.763]$ \\
$A_{5}$ & $\Delta[0.575,0.708]$ & $\Delta[0.688,0.688]$ & $\Delta[0.783,0.821]$ & $\Delta[0.925,0.925]$ & $\Delta[0.813,0.813]$ \\
$A_{6}$ & $\Delta[0.642,0.642]$ & $\Delta[0.500,0.783]$ & $\Delta[0.858,0.858]$ & $\Delta[0.783,0.783]$ & $\Delta[0.738,0.813]$ \\
\hline
\end{tabular}


TABLE 4: Collective ideal supplier.

\begin{tabular}{cccccc}
\hline & $C_{1}$ & $C_{2}$ & $C_{3}$ & $C_{4}$ & $C_{5}$ \\
\hline$\widetilde{r}^{*}$ & $\Delta[0.8,0.9]$ & $\Delta[0.9,1]$ & $\Delta[0.8,0.9]$ & $\Delta[0.9,1]$ & $\Delta[0.8,0.9]$ \\
\hline
\end{tabular}

TABLE 5: Aggregated results 1.

\begin{tabular}{cccccc}
\hline & Max & Min & ITND & ITWD & ITHWD \\
\hline$A_{1}$ & $\Delta[0.375]$ & $\Delta[0.019]$ & $\Delta[0.189]$ & $\Delta[0.208]$ & $\Delta[0.196]$ \\
$A_{2}$ & $\Delta[0.233]$ & $\Delta[0.050]$ & $\Delta[0.106]$ & $\Delta[0.106]$ & $\Delta[0.092]$ \\
$A_{3}$ & $\Delta[0.383]$ & $\Delta[0.104]$ & $\Delta[0.241]$ & $\Delta[0.271]$ & $\Delta[0.273]$ \\
$A_{4}$ & $\Delta[0.200]$ & $\Delta[0.025]$ & $\Delta[0.108]$ & $\Delta[0.116]$ & $\Delta[0.109]$ \\
$A_{5}$ & $\Delta[0.263]$ & $\Delta[0.048]$ & $\Delta[0.124]$ & $\Delta[0.117]$ & $\Delta[0.101]$ \\
$A_{6}$ & $\Delta[0.308]$ & $\Delta[0.050]$ & $\Delta[0.162]$ & $\Delta[0.159]$ & $\Delta[0.145]$ \\
\hline
\end{tabular}

TABLE 6: Aggregated results 2.

\begin{tabular}{cccccc}
\hline & ITOWD & ITOWGD & ITOWHD & ITOWED & ITOWCD \\
\hline$A_{1}$ & $\Delta[0.184]$ & $\Delta[0.131]$ & $\Delta[0.080]$ & $\Delta[0.224]$ & $\Delta[0.252]$ \\
$A_{2}$ & $\Delta[0.094]$ & $\Delta[0.080]$ & $\Delta[0.071]$ & $\Delta[0.111]$ & $\Delta[0.128]$ \\
$A_{3}$ & $\Delta[0.240]$ & $\Delta[0.224]$ & $\Delta[0.208]$ & $\Delta[0.253]$ & $\Delta[0.265]$ \\
$A_{4}$ & $\Delta[0.108]$ & $\Delta[0.091]$ & $\Delta[0.072]$ & $\Delta[0.121]$ & $\Delta[0.130]$ \\
$A_{5}$ & $\Delta[0.111]$ & $\Delta[0.084]$ & $\Delta[0.068]$ & $\Delta[0.140]$ & $\Delta[0.162]$ \\
$A_{6}$ & $\Delta[0.158]$ & $\Delta[0.136]$ & $\Delta[0.115]$ & $\Delta[0.176]$ & $\Delta[0.191]$ \\
\hline
\end{tabular}

5.2. Comparative Discussion. To further evaluate the proposed interval 2-tuple linguistic method, we conduct a comparative analysis with some previous linguistic decision making methods, which include the one based on the uncertain linguistic weighted averaging (ULWA) and the uncertain linguistic hybrid aggregation (ULHA) operators [22], the one based on the uncertain linguistic weighted geometric mean (ULWGM) and the uncertain linguistic hybrid geometric mean (ULHGM) operators [23], and the one based on the uncertain linguistic weighted harmonic mean (ULWHM) and the uncertain linguistic hybrid harmonic mean (ULHHM) operators [24]. The ranking results of the six alternatives derived by using these methods are presented in Table 8 . Note that the linguistic term set $S=$ $\left\{s_{0}, s_{1}, \ldots, s_{6}\right\}$ is used for evaluating the alternatives in the compared methods.

From Table 8, it can be observed that the ranking orders of the alternatives obtained by the methods of Xu [22] and Wei [23] are exactly the same as those determined by proposed approach when the ITND, the ITWD, the ITOWD, the ITOWED, and the ITOWCD operators are applied. Further, the ranking of Park et al.s [24] approach is in line with the proposed method using the ITHWD operator. Thus, the proposed supplier evaluation and selection method is validated. However, compared with the listed methods, the proposed approach using interval 2-tuple linguistic distance operators is more reasonable and flexible for solving supplier selection problems because of the following: (i) It has exact characteristic in linguistic information processing and can effectively avoid the loss and distortion of information in traditional linguistic computational models.

(ii) The linguistic term sets with different granularity of uncertainty can be used by decision makers for assessing alternatives. This enables the decision makers to express their judgments more realistically.

(iii) By using a wide range of distance operators, we can take different potential situations into consideration and provide a more complete picture for supplier evaluation and selection. Thus, it is easier to select the alternative that better fits the interests of the decision maker.

\section{Conclusions}

In this paper, we have developed some interval 2-tuple linguistic distance operators including the interval 2-tuple weighted distance (ITWD), the interval 2-tuple ordered weighted distance (ITOWD), and the interval 2-tuple hybrid weighted distance (ITHWD) operators. These distance operators are very suitable to deal with the decision information represented in interval 2-tuple arguments under multigranular linguistic context. We have given three ways to determine the associated weighting vectors and studied some desired properties of the ITOWD operator. Moreover, further generalizations of the ITOWD operator have been presented by using the generalized and the quasi-arithmetic means. The results are the generalized interval 2-tuple ordered weighted distance (GITOWD) and the quasi-arithmetic interval 2tuple ordered weighted distance (Quasi-ITOWD) operators.

The developed interval 2-tuple linguistic distance operators can be applied in many situations already considered with the distance measures such as in statistics, economics, soft computing, and fuzzy set theory. In this paper, we have applied them to multicriteria group supplier selection with interval 2-tuple linguistic information. In addition, a case example from the healthcare industry has been given to verify the developed method and to demonstrate its practicality and effectiveness. The results showed that this approach provides more complete information for decision making because it can consider a wide range of future scenarios according to the interests of the decision maker.

In the future, we expect to present further extensions to the proposed approach by adding new characteristics such as the use of inducing variables or probabilistic aggregations in the decision process and consider the potential application of the developed interval 2-tuple linguistic distance operator to the problems in other fields.

\section{Competing Interests}

The authors declare that there are no competing interests.

\section{Acknowledgments}

This work was supported by the National Natural Science Foundation of China (nos. 71402090 and 71671125), the 
TABLE 7: Rankings of the alternative suppliers.

\begin{tabular}{llrl}
\hline & \multicolumn{1}{c}{ Ranking } & Ranking \\
\hline Max & $A_{4}>A_{2}>A_{5}>A_{6}>A_{1}>A_{3}$ & ITOWD & $A_{2}>A_{4}>A_{5}>A_{6}>A_{1}>A_{3}$ \\
Min & $A_{1}>A_{4}>A_{5}>A_{6}=A_{2}>A_{3}$ & ITOWGD & $A_{2}>A_{5}>A_{4}>A_{1}>A_{6}>A_{3}$ \\
ITND & $A_{2}>A_{4}>A_{5}>A_{6}>A_{1}>A_{3}$ & ITOWHD & $A_{5}>A_{2}>A_{4}>A_{1}>A_{6}>A_{3}$ \\
ITWD & $A_{2}>A_{4}>A_{5}>A_{6}>A_{1}>A_{3}$ & ITOWED & $A_{2}>A_{4}>A_{5}>A_{6}>A_{1}>A_{3}$ \\
ITHWD & $A_{2}>A_{5}>A_{4}>A_{6}>A_{1}>A_{3}$ & ITOWCD & $A_{2}>A_{4}>A_{5}>A_{6}>A_{1}>A_{3}$ \\
\hline
\end{tabular}

TABLE 8: Ranking comparisons.

\begin{tabular}{lccccc}
\hline & \multicolumn{2}{c}{ Xu's method [22] } & \multicolumn{2}{c}{ Wei's method [23] } & \multicolumn{2}{c}{ Park et al.s method [24] } \\
& $\tilde{r}_{j}$ & Ranking & $\widetilde{r}_{j}$ & Ranking & $\tilde{r}_{j}$ \\
\hline$A_{1}$ & {$\left[s_{4.13}, s_{4.29}\right]$} & 5 & {$\left[s_{3.98}, s_{4.11}\right]$} & 5 & {$\left[s_{3.88}, s_{3.99}\right]$} \\
$A_{2}$ & {$\left[s_{5.00}, s_{5.00}\right]$} & 1 & {$\left[s_{4.85}, s_{4.85}\right]$} & 1 & {$\left[s_{4.82}, s_{4.82}\right]$} \\
$A_{3}$ & {$\left[s_{3.86}, s_{3.98}\right]$} & 6 & {$\left[s_{3.67}, s_{3.76}\right]$} & 6 & {$\left[s_{3.49}, s_{3.56}\right]$} \\
$A_{4}$ & {$\left[s_{4.83}, s_{4.97}\right]$} & 2 & {$\left[s_{4.67}, s_{4.81}\right]$} & 2 & {$\left[s_{4.54}, s_{4.67}\right]$} \\
$A_{5}$ & {$\left[s_{4.80}, s_{4.93}\right]$} & 3 & {$\left[s_{4.61}, s_{4.75}\right]$} & 3 & {$\left[s_{4.55}, s_{4.67}\right]$} \\
$A_{6}$ & {$\left[s_{4.53}, s_{4.72}\right]$} & 4 & {$\left[s_{4.37}, s_{4.59}\right]$} & 4 & {$\left[s_{4.28}, s_{4.50}\right]$} \\
\hline
\end{tabular}

National Social Science Foundation (no. 15CGL003), the Program for Professor of Special Appointment (Young Eastern Scholar) at Shanghai Institutions of Higher Learning (no. QD2015019), and the Shanghai Science and Technology Innovation Action Plan Soft Science Foundation (no. 16692103800).

\section{References}

[1] R. R. Yager, "On ordered weighted averaging aggregation operators in multicriteria decision making," IEEE Transactions on Systems, Man, and Cybernetics, vol. 18, no. 1, pp. 183-190, 1988.

[2] Z. Xu and J. Chen, "Ordered weighted distance measure," Journal of Systems Science and Systems Engineering, vol. 17, no. 4, pp. 432-445, 2008.

[3] J. M. Merigó and A. M. Gil-Lafuente, "New decision-making techniques and their application in the selection of financial products," Information Sciences, vol. 180, no. 11, pp. 2085-2094, 2010.

[4] J. M. Merigó, Y. Xu, and S. Zeng, "Group decision making with distance measures and probabilistic information," KnowledgeBased Systems, vol. 40, pp. 81-87, 2013.

[5] S. Zeng, J. M. Merigó, and W. Su, “The uncertain probabilistic OWA distance operator and its application in group decision making," Applied Mathematical Modelling., vol. 37, no. 9, pp. 6266-6275, 2013.

[6] J. M. Merigó, M. Casanovas, and S. Z. Zeng, "Distance measures with heavy aggregation operators," Applied Mathematical Modelling, vol. 38, no. 13, pp. 3142-3153, 2014.

[7] J. M. Merigó and M. Casanovas, "Decision making with distance measures and linguistic aggregation operators," International Journal of Fuzzy Systems, vol. 12, no. 3, pp. 190-198, 2010.

[8] S. Zeng and W. Su, "Intuitionistic fuzzy ordered weighted distance operator," Knowledge-Based Systems, vol. 24, no. 8, pp. 1224-1232, 2011.

[9] S. Zeng, "Some Intuitionistic Fuzzy Weighted Distance Measures and Their Application to Group Decision Making," Group Decision and Negotiation, vol. 22, no. 2, pp. 281-298, 2013.
[10] Z. S. Xu, "Fuzzy ordered distance measures," Fuzzy Optimization and Decision Making, vol. 11, no. 1, pp. 73-97, 2012.

[11] S. Xian and W. Sun, "Fuzzy linguistic induced Euclidean OWA distance operator and its application in group linguistic decision making," International Journal of Intelligent Systems, vol. 29, no. 5, pp. 478-491, 2014.

[12] W. Su, C. Zhang, and S. Zeng, "Uncertain induced heavy aggregation distance operator and its application to decision making," Cybernetics and Systems, vol. 46, no. 3-4, pp. 172-187, 2015.

[13] L. Zhou, F. Jin, H. Chen, and J. Liu, "Continuous intuitionistic fuzzy ordered weighted distance measure and its application to group decision making," Technological and Economic Development of Economy, vol. 22, no. 1, pp. 75-99, 2016.

[14] S. Zeng, J. Chen, and X. Li, "A hybrid method for pythagorean fuzzy multiple-criteria decision making," International Journal of Information Technology \& Decision Making, vol. 15, no. 02, pp. 403-422, 2016.

[15] S. Xian, W. Sun, S. Xu, and Y. Gao, "Fuzzy linguistic induced OWA Minkowski distance operator and its application in group decision making," PAA. Pattern Analysis and Applications, vol. 19, no. 2, pp. 325-335, 2016.

[16] H. Cheng, F. Meng, and K. Chen, "Several generalized intervalvalued 2-tuple linguistic weighted distance measures and their application," International Journal of Fuzzy Systems, 2016.

[17] F. Meng and X. Chen, "A hesitant fuzzy linguistic multigranularity decision making model based on distance measures," Journal of Intelligent and Fuzzy Systems, vol. 28, no. 4, pp. 1519-1531, 2015.

[18] J. M. Merigó and M. Casanovas, "Decision-making with distance measures and induced aggregation operators," Computers \& Industrial Engineering, vol. 60, no. 1, pp. 66-76, 2011.

[19] H. Zhang and L. Yu, "New distance measures between intuitionistic fuzzy sets and interval-valued fuzzy sets," Information Sciences, vol. 245, pp. 181-196, 2013.

[20] M. Düğenci, "A new distance measure for interval valued intuitionistic fuzzy sets and its application to group decision making problems with incomplete weights information," Applied Soft Computing Journal, vol. 41, pp. 120-134, 2016. 
[21] C. Lu, J. X. You, H. C. Liu, and P. Li, "Health-care waste treatment technology selection using the interval 2-tuple induced TOPSIS method," International Journal of Environmental Research and Public Health, vol. 13, no. 6, p. 562, 2016.

[22] Z. S. Xu, "Uncertain linguistic aggregation operators based approach to multiple attribute group decision making under uncertain linguistic environment," Information Sciences, vol. 168, no. 1-4, pp. 171-184, 2004.

[23] G.-W. Wei, "Uncertain linguistic hybrid geometric mean operator and its application to group decision making under uncertain linguistic environment," International Journal of Uncertainty, Fuzziness and Knowledge-Based Systems, vol. 17, no. 2, pp. 251-267, 2009.

[24] J. H. Park, M. G. Gwak, and Y. C. Kwun, "Uncertain linguistic harmonic mean operators and their applications to multiple attribute group decision making," Computing, vol. 93, no. 1, pp. 47-64, 2011.

[25] H. Zhang, "Some interval-valued 2-tuple linguistic aggregation operators and application in multiattribute group decision making," Applied Mathematical Modelling, vol. 37, no. 6, pp. 4269-4282, 2013.

[26] J.-Q. Wang, D.-D. Wang, H.-Y. Zhang, and X.-H. Chen, "Multicriteria group decision making method based on interval 2tuple linguistic information and Choquet integral aggregation operators," Soft Computing, vol. 19, no. 2, pp. 389-405, 2015.

[27] A. Singh, A. Gupta, and A. Mehra, "Energy planning problems with interval-valued 2-tuple linguistic information," Operational Research, 2016.

[28] H.-C. Liu, J.-X. You, and X.-Y. You, "Evaluating the risk of healthcare failure modes using interval 2-tuple hybrid weighted distance measure," Computers \& Industrial Engineering, vol. 78, pp. 249-258, 2014.

[29] M. Shan, J. You, and H. Liu, "Some interval 2-tuple linguistic harmonic mean operators and their application in material selection," Advances in Materials Science and Engineering, vol. 2016, Article ID 7034938, 13 pages, 2016.

[30] F. Meng, M. Zhu, and X. Chen, "Some generalized intervalvalued 2-tuple linguistic correlated aggregation operators and their application in decision making," Informatica, vol. 27, no. 1, pp. 111-139, 2016.

[31] F. Herrera and L. Martínez, "A 2-tuple fuzzy linguistic representation model for computing with words," IEEE Transactions on Fuzzy Systems, vol. 8, no. 6, pp. 746-752, 2000.

[32] W.-S. Tai and C.-T. Chen, "A new evaluation model for intellectual capital based on computing with linguistic variable," Expert Systems with Applications, vol. 36, no. 2, pp. 3483-3488, 2009.

[33] F. Herrera and L. Martínez, "A model based on linguistic 2tuples for dealing with multigranular hierarchical linguistic contexts in multi-expert decision-making," IEEE Transactions on Systems, Man, and Cybernetics Part B: Cybernetics, vol. 31, no. 2, pp. 227-234, 2001.

[34] H. Zhang, "The multiattribute group decision making method based on aggregation operators with interval-valued 2-tuple linguistic information," Mathematical and Computer Modelling, vol. 56, no. 1-2, pp. 27-35, 2012.

[35] X. Liu, Z. Tao, H. Chen, and L. Zhou, "A new interval-valued 2-tuple linguistic bonferroni mean operator and its application to multiattribute group decision making," International Journal of Fuzzy Systems, 2016.

[36] Q. Wu, P. Wu, Y. Zhou, L. Zhou, H. Chen, and X. Ma, "Some 2-tuple linguistic generalized power aggregation operators and their applications to multiple attribute group decision making," Journal of Intelligent and Fuzzy Systems, vol. 29, no. 1, pp. 423436, 2015.

[37] H. C. Liu, J. X. You, P. Li, and Q. Su, "Failure mode and effect analysis under uncertainty: an integrated multiple criteria decision making approach," IEEE Transactions on Reliability, vol. 65, no. 3, pp. 1380-1392, 2016.

[38] H. Liu, J. You, S. Chen, and Y. Chen, "An integrated failure mode and effect analysis approach for accurate risk assessment under uncertainty," IIE Transactions, vol. 48, no. 11, pp. 10271042, 2016.

[39] L. Martínez and F. Herrera, "An overview on the 2-tuple linguistic model for computing with words in decision making: extensions, applications and challenges," Information Sciences, vol. 207, pp. 1-18, 2012.

[40] Z. Xu, Uncertain Multi-Attribute Decision Making: Methods and Applications, Springer, New York, NY, USA, 2014.

[41] G.-W. Wei, "Extension of TOPSIS method for 2-tuple linguistic multiple attribute group decision making with incomplete weight information," Knowledge and Information Systems, vol. 25, no. 3, pp. 623-634, 2010.

[42] Z. Xu, "An overview of methods for determining OWA weights," International Journal of Intelligent Systems, vol. 20, no. 8, pp. 843-865, 2005.

[43] G. Yari and A. R. Chaji, "Maximum Bayesian entropy method for determining ordered weighted averaging operator weights," Computers \& Industrial Engineering, vol. 63, no. 1, pp. 338-342, 2012.

[44] B. S. Ahn and H. Park, "Least-squared ordered weighted averaging operator weights," International Journal of Intelligent Systems, vol. 23, no. 1, pp. 33-49, 2008.

[45] Z. S. Xu and M. M. Xia, "Distance and similarity measures for hesitant fuzzy sets," Information Sciences, vol. 181, no. 11, pp. 2128-2138, 2011.

[46] R. R. Yager, "Generalized OWA aggregation operators," Fuzzy Optimization and Decision Making, vol. 3, no. 1, pp. 93-107, 2004.

[47] J. Q. Wang, J. T. Wu, J. Wang, H. Y. Zhang, and X. H. Chen, "Multi-criteria decision-making methods based on the Hausdorff distance of hesitant fuzzy linguistic numbers," Soft Computing, vol. 20, no. 4, pp. 1621-1633, 2016.

[48] L. A. Zadeh, "The concept of a linguistic variable and its application to approximate reasoning. I," Information Sciences, vol. 8, no. 3, pp. 199-249, 1975.

[49] Z. Xu, "A method based on linguistic aggregation operators for group decision making with linguistic preference relations," Information Sciences, vol. 166, no. 1-4, pp. 19-30, 2004. 


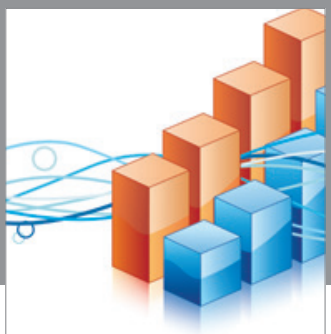

Advances in

Operations Research

vatem alat4

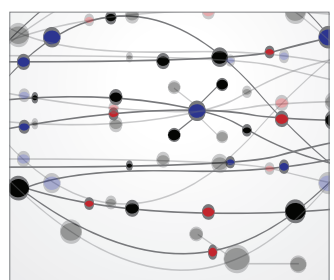

\section{The Scientific} World Journal
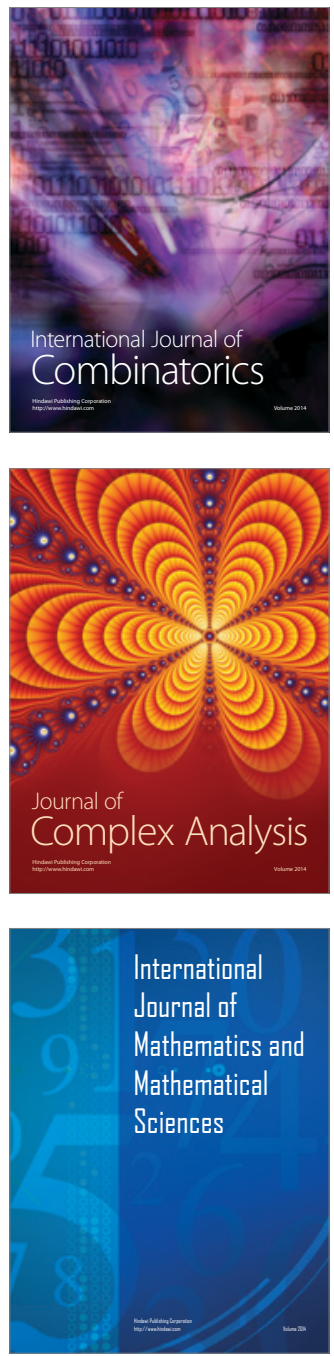
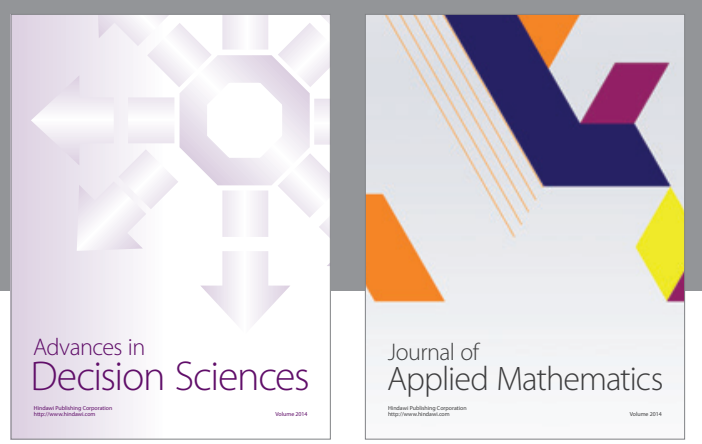

Algebra

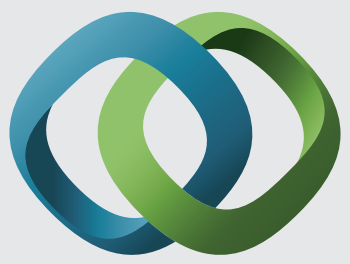

\section{Hindawi}

Submit your manuscripts at

http://www.hindawi.com
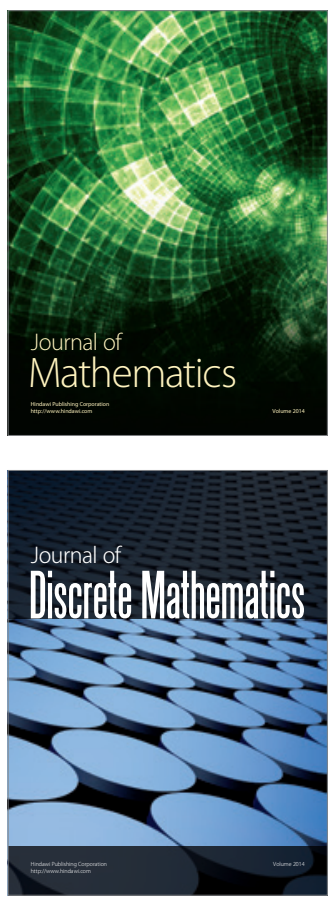

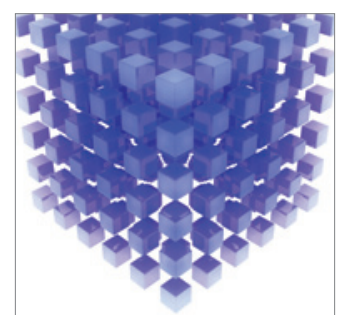

Mathematical Problems in Engineering
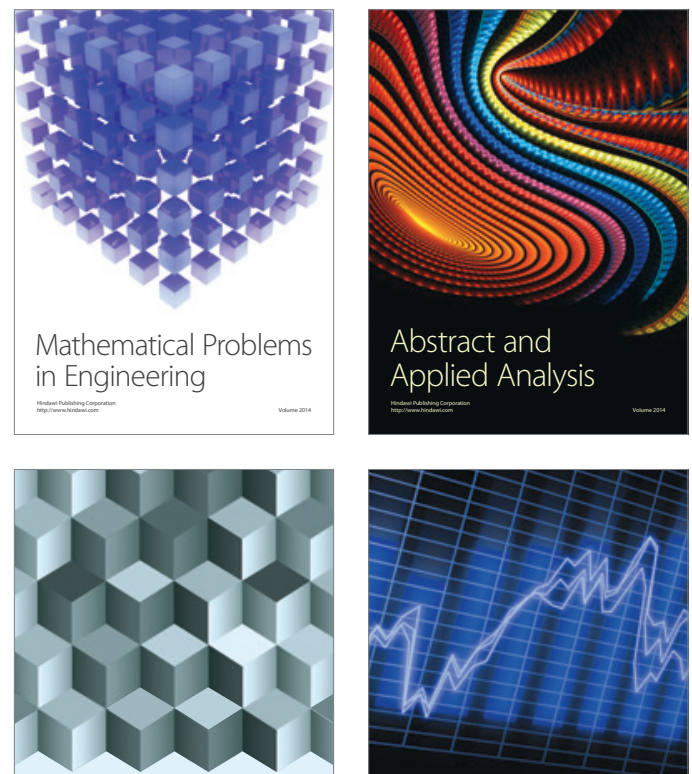

Journal of

Function Spaces

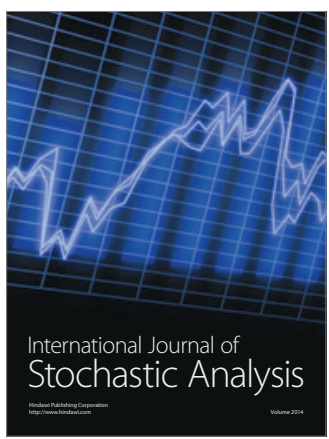

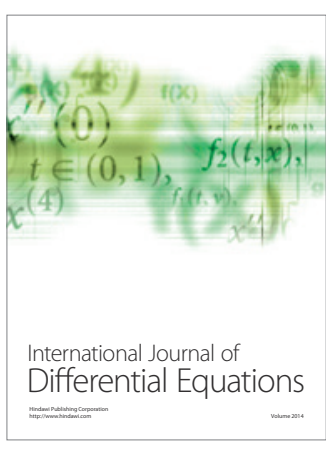
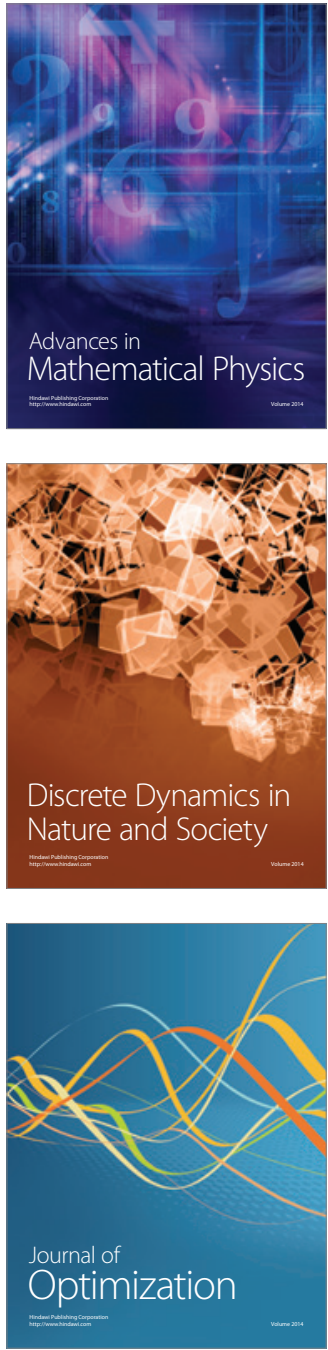